\title{
Towards chemistry at absolute zero
}

\author{
Brianna R. Heazlewood ${ }^{1, *}$ and Timothy P. Softley ${ }^{2, *}$ \\ ${ }^{1}$ Department of Chemistry, Physical and Theoretical Chemistry Laboratory, South Parks Road, Oxford, OX1 3QZ, \\ United Kingdom \\ ${ }^{2}$ University of Birmingham, Edgbaston, B15 2TT, United Kingdom \\ *e-mail: brianna.heazlewood@chem.ox.ac.uk; t.p.softley@bham.ac.uk
}

\section{ABSTRACT}

The prospect of cooling matter down to temperatures that are close to the absolute zero raises intriguing questions about how chemical reactivity changes under these extreme conditions. Although some types of chemical reaction still occur at $1 \mu \mathrm{K}$, they can no longer adhere to the conventional picture of reactants passing over an activation energy barrier to become products. Indeed at ultracold temperatures, the system enters a fully quantum regime, and quantum mechanics replaces the classical picture of colliding particles.

In this Review, we discuss recent experimental and theoretical developments that allow us to explore chemical reactions at temperatures that range from $10 \mathrm{~K}$ to $500 \mathrm{nK}$. Although the field is still in its infancy, exceptional control has already been demonstrated over reactivity at low temperatures.

\section{Introduction}

The temperature dependence of chemical reaction rates is a crucial concept for virtually all applications of chemistry. It is key to understanding and controlling the mechanisms and product branching ratios of chemical processes. A number of models that predict the temperature dependence of bimolecular reactions, including transition state theory and collision theory, are based on the equation proposed by Arrhenius in $1889^{1}$. According to the Arrhenius equation, $k=A \mathrm{e}^{-E_{a} / R T}$ (where $k$ is the rate coefficient, $A$ is the pre-exponential factor, $E_{a}$ is the activation energy, $R$ is the gas constant and $T$ is temperature), the rate coefficient is determined by a statistical fraction of chemical collisions in a sample that have sufficient energy to overcome the activation barrier. It follows that the rates of simple bimolecular chemical processes should increase dramatically with increasing temperature, and reactions at very low temperatures would proceed extremely slowly and ultimately occur to a negligible extent.

Although the Arrhenius equation remains broadly valid, there are two reasons why it may not always be applicable in the regime of ultracold chemistry. First, there are certain types of exothermic chemical reactions that typically have no energetic barrier. Examples of such barrier-less exothermic reactions include those occurring between free-radicals, between ionic and neutral species, and those involving electronically excited molecules. Barrier-less reactions are prevalent in the interstellar medium, with ions and radicals readily created from the photoionization and photodissociation of more stable species by short-wavelength radiation. For $E_{a}=0$, the Arrhenius equation would predict $k=A$, a rate constant that either does not vary with temperature (if $A$ is genuinely temperature independent), or varies slowly with temperature. $E_{a}=0$ also implies very fast reactions, as exemplified by reactions involving either ions or radicals in the interstellar medium. As we explain in this Review, however, the Arrhenius equation does not really apply in these circumstances.

The second, and perhaps more intriguing, reason why the Arrhenius equation cannot be applicable to ultracold chemistry is associated with the transition from the classical to the quantum regime that occurs at very low temperatures. The concept of wave-particle duality is neatly expressed by the deBroglie equation, $p=h / \lambda$, which relates the momentum of a particle $(p)$ to the wavelength $(\lambda)$ of the quantum mechanical wave describing its motion. As the temperature is lowered, the momentum of particles decreases and the associated wavelength increases. At room temperature, the wavelength associated with the translational motion of small molecules, such as water or ammonia, is one order of magnitude smaller than typical chemical dimensions $(\sim 0.1 \AA)$. Under these conditions, collisions of these small molecules can essentially be described as classical with the particle-like behaviour dominating over the wave-like — and we can picture the passage of the system through the activation barrier as a ball rolling over a hill. The relevant 'chemical dimension' here is the width of the activation barrier. In the potential energy profile that is commonly used to represent a reaction, the reaction co-ordinate has a physical dimension of length, and the width of the reaction barrier is of the order of a chemical bond length. If the temperature of a system is lowered by four orders of magnitude (for example, from $300 \mathrm{~K}$ to $0.03 \mathrm{~K}$ ), the translational wavelength increases by two orders of magnitude and for small molecules it becomes one order of magnitude larger $(\sim 1 \mathrm{~nm})$ than the width of the barrier. At 
ultracold temperatures, the classical description of the collision of particles needs to be replaced with a quantum description and the picture of a ball rolling over a hill needs to be replaced by that of a wave propagating across a barrier.

The quantum regime is a physical regime in which our basic understanding of how chemical processes happen, in terms of molecules bumping into each other and breaking and remaking bonds, needs to be challenged. Strictly speaking, the dynamical system of moving and colliding molecules needs to be described as a superposition of partial waves, the components of which represent the different angular momentum states associated with the relative motion of the colliding particles. As the temperature is lowered, the system moves towards a regime in which it can be described by just a few quantised collisional angular momentum states (and, ultimately, just a single state). We denote the collisions as ' $s$-wave' or ' $p$-wave' collisions (known as the partial-wave description) to represent those with zero or one unit of collisional angular momentum, respectively. Furthermore, in the quantum regime the population of molecular quantum states is distributed over just a few rovibrational states and ultimately collapses to a single quantum state at the lowest temperatures (under conditions of thermal equilibrium). At low temperatures, quantum effects on the rate coefficient and other properties of the reaction, such as collision energy resonances, quantum reflection and geometric phase (described in detail in the 'Reactions in the ultracold regime' section), are most likely to be observable. This is in contrast to what we observe at room temperature, where we understand the overall rate of a process to be an average of the behaviours of individual collisions of molecules with vastly varying sets of quantum numbers and a distribution of energies.

When the temperature reaches sub- $\mu \mathrm{K}$, the translational wavelength typically exceeds the average separation of molecules in the sample, even for a low density gas, and ultimately the system may enter the regime of quantum degeneracy (for example, bosonic particles that form a Bose-Einstein condensate, in which all particles are in the same quantum state and the density distribution of the gas sample is described by a single overarching macroscopic wave). In this case, the collision of any one molecule cannot be considered independently from the collision of another in the sample, and we expect a 'coherence' (analogous to that observed in laser radiation) between the translational waves of the individual gas molecules. Under these circumstances, many-body quantum effects would need to be considered in order to accurately describe the behaviour of the system.

Although the ultracold regime is completely artificially created in the laboratory, given the lowest temperatures in the natural universe are associated with the cosmic microwave background at $2.725 \mathrm{~K}^{5}$, ultracold conditions provide an exciting and extreme physical regime for studying chemical reactivity. Under these conditions, we explore how chemistry might occur differently and possibly be brought under a high level of control, and can gain new insights into the role of quantum mechanics in chemical reactivity. The 20th century has seen remarkable advances in the development of techniques for creating low-density gas samples in the laboratory at ultracold temperatures. Laser cooling was developed in the 1970s, with the importance of the technique recognised by the 1997 Physics Nobel Prize ${ }^{3}$. Laser cooling has enabled the trapping of atoms, and subsequently molecules, at temperatures below $1 \mu \mathrm{K}\left(10^{-6} \mathrm{~K}\right)$. It has been accompanied by and combined with an abundance of other techniques, such that a wide range of chemical species can now be cooled to low temperatures ${ }^{4}$.

The field of astrophysical chemistry has sought to understand the chemical composition of the interstellar medium through developing reaction models for the complex processes that occur at the low temperatures of interstellar clouds — typically $10-50 \mathrm{~K}$. The field has its origins in astronomical observations, and the numbers of complex molecular species detected spectroscopically by radiofrequency and optical telescopes is ever increasing ${ }^{2}$. Tracing the origins of interstellar species requires a detailed understanding of the chemistry occurring within very low density gas samples, on extremely long timescales and at low temperatures. The development of methods to reach such low temperatures in the laboratory and measure chemical reaction rates and products under these conditions has driven considerable growth of the field.

In this Review article, we discuss recent progress in the study of cold $(>1 \mathrm{mK})$ and ultracold $(<1 \mathrm{mK})$ chemistry, focusing on the most exciting and chemically interesting advances in the field. We show that not only can chemical reactions be observed at temperatures very close to the absolute zero $(0 \mathrm{~K})$, they can also display new features, including quantum effects (such as tunnelling, quantum reflection and quantisation of collisional angular momentum) and long-lived intermediate complexes. We also discuss the exciting prospect under these conditions to control all parameters of a bimolecular encounter using optical, electric and magnetic fields.

\section{Key experimental methodologies}

A range of complementary methods has been developed over several decades for the study of chemistry under cold (>1 mK) and ultracold $(<1 \mathrm{mK})$ conditions in low-density gases (in most cases under ultra-high vacuum conditions). It is worth noting here that the terms 'cold' and 'ultracold' can sometimes be defined differently by different communities. In atomic and molecular physics, for example, the term 'ultracold' typically refers to collisions that occur under single-partial-wave conditions at approximately $1 \mu \mathrm{K}$. Key recent experimental developments include deceleration and trapping of molecules by electromagnetic and optical fields, laser cooling of molecules and magnetoassociation of laser-cooled atoms, merged beam experiments, ion trapping, cooling by collisions (sympathetic, evaporative and buffer-gas cooling), and velocity filtering. All these approaches 
and the relevant references are discussed in Boxes 1-5, with the specific applications of these methods detailed in the following sections. For many of these methods, samples are not produced at true thermal equilibrium and, therefore, the concept of a temperature is not always rigorously applicable. As is conventional in this field, we use temperature as a measure of the mean energy in the different degrees of freedom, and different degrees of freedom (dof) may be categorised by different 'temperatures' $\left(E_{\mathrm{dof}}=k T_{\mathrm{dof}}\right)$. In that sense, chemistry at low temperatures is really chemistry at low energies, and experimentally it requires a large degree of control over the energy states of the different dof of the reactants.

Given that the collision energy only depends on the relative motion of the colliding reactants, it can either be controlled by constraining the relative velocities of reactants in a moving frame of reference (see Box 1 and references therein) or by manipulating their absolute velocities (Boxes 2-5). For $T>1 \mathrm{mK}$, either approach can be used and a wide range of chemical systems can be studied. The relative velocity approach generally involves the use of supersonic beams, in which pairs of reactant beams are crossed at a narrow angle or merged onto a single axis, such that the reactants have low relative velocities (Box 1). In the cold regime, the absolute velocity approach involves a variety of methodologies that typically rely on electromagnetic or optical fields to manipulate the properties of selected species of interest (Box 2), with cooling and trapping of ionic species requiring very different experimental setups to those used for neutral species (Box 3). Molecular beams can be decelerated and the slow-moving target species subsequently trapped (Box 2). Velocity-selection or trapping can also be combined with buffer-gas cooling methods (Box 4). Achieving ultracold temperatures (below $1 \mathrm{mK}$ ) generally requires the combination of laser cooling and trapping methods (Box 5), which can currently only be applied to a very limited range of chemical species. We are not aware of any measurements undertaken on a single chemical reaction system that spans both the cold and ultracold regimes.

The study of wide-ranging classes of chemical reactions at extremely low temperatures generally requires the simultaneous production and cooling of more than one reactant. Many of the methods and the corresponding existing experimental setups for producing cold or ultracold species (Boxes 1-5) are tailored to the production of one (or a narrow range of) specific chemical species. The specific molecular properties of the species of interest, such as spectroscopic transitions, polarisability and electric or magnetic dipole moment, require system-specific tuning of the electromagnetic or optical fields. For example, the Stark deceleration of a beam of $\mathrm{ND}_{3}$ to near-zero velocity requires a decelerator with around 65 electrode pairs, whereas the deceleration of NO requires over 1100 electrode pairs, as a consequence of the very different dipole moments of these species $^{32}$. It is a significant experimental challenge to combine more than one technique to bring together different species at an equivalent temperature. For example, examining cold collisions between ionic species and neutral species usually involves different types of cooling processes. When co-trapping ionic and neutral species is necessary, there must be a superposition of the neutral and ionic trapping fields. An additional factor to consider is that for exothermic processes, the products will take away the excess energy of the collision process and will not necessarily be cold (or able to be cooled) under the experimental conditions, making it challenging to monitor product formation directly.

\section{Theoretical approaches}

We noted in the Introduction that the fastest chemical reactions in the cold and ultracold regime tend to exhibit negligible reaction barriers. For many years, these fast reactions have been shown to be amenable to the application of capture theory. In simple terms, capture theory — which in its classical form can be attributed to Langevin, amongst others — assumes that the probability of a reaction is determined by the probability of the reactants in a given collision to be captured irreversibly into a reaction complex. The capture is the rate-determining step in these cases. Once the complex is formed, the reaction is assumed to proceed to products with $100 \%$ probability. The reactants are drawn together into the complex, which corresponds to a well in the reaction potential energy surface, by long-range attractive forces. Strong long-range forces (as occur in collisions between ions and dipolar species) give rise to large reaction cross-sections and high rates. However, the formation of the complex, and the overall reaction, can be hindered by the angular momentum of the reacting pair of molecules. The angular momentum is determined by the relative motion of the reactant species, hence by their relative velocity, and the distance of closest approach (the impact parameter). The angular momentum is responsible for an effective, centrifugal barrier in the potential energy profile of the reaction. Therefore, the reaction probability depends on collision energy, which in turn depends on the exact nature of the long-range forces and the probability that a given collision surmounts the centrifugal barrier.

The purely classical version of capture theory, described in more detail in Ref. 73, has successfully predicted the rate coefficients of many fast reactions over a wide temperature range, from $\sim 10 \mathrm{~K}$ to above $300 \mathrm{~K}$. The methodology can also be adapted to account for the rotational-quantum-state-dependence of the effective reaction energy profile, as incorporated into the rotationally adiabatic capture theory, or the statistical adiabatic channel model ${ }^{74-76}$. Explicitly considering the reactivity of individual rotational states of the reactant molecules becomes important at low temperatures - where only a few levels and ultimately a single quantum state are populated — as it is predicted that molecules with zero rotational angular momentum can behave very differently from those with a few quanta of excitation ${ }^{75}$.

Capture methods can be extended to the lowest of temperatures by adopting a fully quantum version ${ }^{77}$. In this case, 
the propagation of a quantum wave across the effective centrifugal barrier is explicitly calculated. This wave describes the relative position and motion of the atoms in the colliding pair of molecules. Its propagation involves numerical integration of the Schrödinger equation for motion on the appropriate potential energy surface, with an absorbing boundary condition at some short reactant separation inside the potential well region of complex formation. This condition imposes a decay of the wavefunction to zero over a finite distance eliminating non-physical artefacts in the calculation ${ }^{78}$. The rate coefficient is derived from the probability density of the wave in the reactant (pre-barrier) region and at the well region. By comparing quantum and classical calculations of the barrier penetration probability, an effective transition temperature can be defined at which the quantum results begin to deviate from the classical prediction. For the reactions of neutral polar species (dipole-dipole collisions), in which the rotationally averaged long-range potential varies as $r^{-6}$ (with $r$ the internuclear separation), a typical transition temperature is predicted in the $\mathrm{mK}$ regime ${ }^{77}$. For ion-neutral (non-polar) collisions, where the long range potential varies as $r^{-4}$, the transition temperature is predicted in the $\mu \mathrm{K}$ regime ${ }^{77}$. However, because very few measurements of ion-neutral collisions have been made in the ultracold regime, it is likely that the true quantum regime has not been fully probed to date for collisions of this type. The principal effect that causes deviation from classical capture theory in the case of dipole-dipole collisions is quantum reflection of the wave at the point of the potential drop - just as a wave can penetrate an energy barrier on a potential surface, it can also be reflected from a dip, preventing complex formation and thus hindering reaction. The steeper the potential drop caused by the intermolecular attraction, the stronger the quantum reflection effect and the higher the temperature at which it leads to a deviation from classical predictions. For ion-neutral collisions, the smaller quantum reflection effect is counterbalanced by tunnelling through the centrifugal barrier, except at much lower temperatures ${ }^{77}$.

Capture methods can only predict a total cross-section for reaction - the probability of any reaction occurring, irrespective of the detailed outcome of the reaction. The product branching ratio (where more than one chemical pathway is open) or the product quantum state distributions cannot be predicted by capture theories. This is because the properties of PESs in the product region are not explicitly considered, and therefore the partitioning of the system on these surfaces cannot be accounted for. To calculate partial reaction cross-sections for reactions, and hence the relative probabilities for producing different product channels, a full quantum dynamics approach needs to be adopted. This requires the accurate calculation of all PESs involved in the reaction, followed by a quantum wave propagation of trajectories over the surfaces, or equivalently solving the time-independent Schrödinger equation for a multichannel wavefunction (the coupled channel approach) ${ }^{79}$.

The use of a full quantum dynamics approach to study reactivity at ultracold temperatures poses some serious technical challenges. In this regime, the influence of long-range forces requires PESs to be calculated accurately over an extensive region. At the same time, it is necessary to accurately calculate the details of the potential well corresponding to complex formation. Furthermore, fast reactions involving ions or radicals typically require unpaired electron spin and sometimes electronic orbital momentum to be considered explicitly in the calculations. This, in turn, frequently requires spin-orbit coupling and multiple PESs to be included, which means accounting for the possibility of non-adiabatic transitions between these surfaces. Deep potential wells associated with intermediate complex formation also cause immense challenges for quantum dynamics in some reaction systems, such as those featuring heavy alkali-metals that have been studied experimentally ${ }^{155}$. The large number of quantum states involved in these systems requires the use of multichannel wavefunctions with basis-sets that are too large to be handled in the quantum dynamics coupled-channel calculations. Some success has been achieved by using approaches based on quantum defect theory. These approaches use a single short-range parameter to account for short-range multichannel interactions in the specific reacting system, while longer range interactions are included analytically (rather than numerically) using formulae that are determined by molecular properties such as the dipole moment and polarisibility ${ }^{80,81}$. It is the short range interactions within the reaction complex that govern the detailed outcome of the reaction while the longer range interactions govern the rate of capture.

A further disadvantage of the quantum capture approach is that the application of the absorbing boundary condition eliminates resonant features of the scattering process. Resonances have been experimentally observed in the low energy collisions of some simple species (such as the $\mathrm{F}+\mathrm{H}_{2}, \mathrm{~S}+\mathrm{H}_{2}$ and $\mathrm{He}^{*}+\mathrm{H}_{2}$ reactions discussed below), appearing as peaks in the plot of the cross-section of the reaction as a function of collision energy ${ }^{82-87}$. They represent the quantised states of the collision complex and are associated with the rotation of the colliding species around one another or the intermolecular vibrations within the complex. The collision energies at which resonances occur and the widths of the resonant peaks, which are related to the increased lifetime of the complex when captured into one of these states, can be predicted using a full quantum dynamics approach. However, such calculations are extremely sensitive to the accuracy of the PESs in the short-range region, and the non-adiabatic interactions (that is, the coupling between different potential energy surfaces) need to be considered over a wide range of geometries. Also, because resonance states are not fully-bound quantum states, but rather quasi-bound, their energy varies strongly with the collisional angular momentum. Hence, the prediction of the energies at which resonances are positioned is much more challenging than, for example, calculating the energies of bound molecules of a similar size. Calculating the location and width of quantum resonances is acknowledged as an extreme test of the accuracy of quantum chemistry calculations ${ }^{88,89}$. 


\section{Reactions in the astrophysical temperature regime}

We start our journey towards absolute zero by considering reactions that occur under conditions comparable to those found in the coldest parts of the Universe. In the interstellar medium (ISM), dense molecular clouds typically exhibit temperatures on the order of 10-20 K. Star-forming components of the ISM are somewhat warmer, with temperatures spanning 50-100 K. At temperatures of 10-100 K, bimolecular reactions exhibiting significant energy barriers tend to proceed at negligible rates, although tunnelling of $\mathrm{H}$ or other light atoms becomes increasingly important at low temperatures ${ }^{91}$. Some reactions can exhibit complex behaviour as a function of the temperature, for example showing a maximum or minimum in the rate coefficient and quantum resonance effects at the lower end of the temperature regime ${ }^{92,93}$. Fast barrierless reactions involving radical species and ions can be usually described using classical capture models, although there are already some notable exceptions to this rule, as discussed in the following paragraphs.

Rate coefficients of ion-polar-molecule reactions including $\mathrm{N}^{+}+\mathrm{H}_{2} \mathrm{O}$ and $\mathrm{N}^{+}+\mathrm{NH}_{3}$ have been shown to exhibit an inverse temperature dependence. In other words, the rate coefficient increases as the temperature decreases ${ }^{90,94,95}$. The results of reaction kinetics in uniform supersonic flow (CRESU) experiments have also challenged the long-held view that reactions featuring an activation barrier are unimportant in the chemistry of dense interstellar clouds (Box 1). The $\mathrm{OH}+\mathrm{CH}_{3} \mathrm{OH}$ reaction, which exhibits a significant activation energy barrier, was found to proceed through a hydrogen bonded complex that could tunnel through the barrier to products. The rate coefficient for this reaction was found to be almost two orders of magnitude higher at $60 \mathrm{~K}$ than at $200 \mathrm{~K}$, a surprising result for a reaction with an energetic barrier ${ }^{96}$. A similar result has been seen in the reaction of $\mathrm{F}$ with $\mathrm{H}_{2}$ at low temperatures ${ }^{97}$ for which the rate coefficient has been measured over a range $10-100 \mathrm{~K}$ with a minimum at around $20 \mathrm{~K}$ that increased at lower temperatures. As these examples illustrate, rate coefficients do not always display a monotonic trend with temperature. Rate coefficients can be enhanced as a result of quantum behaviour such as tunnelling through a barrier or subtle features on the underlying PES(s), influencing the likelihood of a reaction to occur at a given collision energy. The presence of long-lived, weakly-bound intermediates can also give rise to rate coefficients that display unusual temperature dependencies. In the case of $\mathrm{OH}+\mathrm{CH}_{3} \mathrm{OH}$, the lifetime of the intermediate complex increases with decreasing temperature, enhancing the opportunity for the system to tunnel through the reaction barrier to form products. This tunneling results in the high rate coefficient observed at low temperatures. For the $\mathrm{F}+\mathrm{H}_{2}$ reaction, tunnelling also plays a strong role at the lowest temperatures. This process is of astrochemical interest, as it is the only known source of HF in interstellar clouds. The accurate calculation of the temperature dependence of the reaction-rate requires explicit inclusion of spin-orbit coupling of the $\mathrm{F}$ atom, which leads to a splitting of the lowest potential energy surface, and of the resonance effects at low temperatures (see below) ${ }^{97}$.

Reaction pathways that may play a role in the formation of larger carbocations in the ISM and other extraterrestrial environments have also been examined in a recent study. The $\mathrm{CCl}^{+}+\mathrm{C}_{2} \mathrm{H}_{2}$ reaction system was monitored in a Coulomb crystal (see Box 3 for an explanation of how reactions are monitored in Coulomb crystals), with the experimental rate coefficients in agreement with predictions from classical Langevin capture theory ${ }^{98}$. Astrochemically-relevant reactions between ions and neutral species can be studied in 22-pole ion traps (see Box 3) over an extended temperature range. For example, rate coefficients for the reaction $\mathrm{CH}^{+}+\mathrm{H} \rightarrow \mathrm{C}^{+}+\mathrm{H}_{2}$ have been measured over the temperature range 12-100 K using a cryogenic 22-pole trap apparatus combined with a beam of cold $\mathrm{H}$ atoms. The rate coefficient displays an unusual temperature dependence, peaking at $60 \mathrm{~K}$ and falling by more than an order of magnitude at $12 \mathrm{~K}^{99}$. While the cause of this behaviour is yet to be confirmed, a preliminary analysis revealed that only the lowest rotational state, $J=0$, which is the least reactive state for $\mathrm{CH}^{+}$, is populated under the coldest conditions. It seems likely that the temperature dependence of the reaction rate could be explained using a rotationally adiabatic version of capture theory that explicitly calculates the detailed variation of the rate with rotational quantum state. The properties of a number of other small ion-molecule systems have been studied in multipole radio-frequency ion traps (Box 3), with some recent examples including the collisions of $\mathrm{OH}^{-}$and $\mathrm{OD}^{-}$with $\mathrm{He}^{101}$, the reactions of $\mathrm{OH}^{+102}, \mathrm{H}_{2} \mathrm{O}^{+102}$ and $\mathrm{OD}^{-}$with $\mathrm{H}_{2}$, and the reaction of $\mathrm{OH}^{-}$with $\mathrm{D}_{2}{ }^{100}$. Low-energy neutral $\mathrm{H}_{2} \mathrm{O}+\mathrm{H}_{2}$ collisions have recently been studied under astrophysical conditions using a crossed beam set-up, with the experimental results in good agreement with quantum theory predictions ${ }^{103}$. In this case, the collisions at very low temperatures lead to energy exchange between the rotations of the two species (rotationally inelastic collisions) rather than reaction, but the temperature dependence of the inelastic cross-section shows resonances associated with the $\mathrm{H}_{2} \mathrm{O}-\mathrm{H}_{2}$ complex that is formed in a fairly deep well $\left(235 \mathrm{~cm}^{-1}\right)$. The correct prediction of the resonances requires quantum dynamics calculations of the very highest accuracy, which is possible for these light-atom systems.

Deuterium fractionation is a phenomenon that is uniquely observed in the low-temperature regions of the ISM. Here, the relative abundance of deuterated molecular species compared to their hydrogenated analogues is well above that predicted from the cosmic D:H ratio ${ }^{104,105}$ using models that incorporate the current understanding of gas phase interstellar chemistry. While the interplay between gas-phase and grain-surface reactions is also expected to be important, the grain-surface interactions are not well characterised currently, and no current models of interstellar chemistry incorporating these can account for the known abundances of deuterated molecular species in the ISM. A recent study examined the charge transfer between $\mathrm{Xe}^{+}$ions 
and both $\mathrm{NH}_{3}$ and $\mathrm{ND}_{3}$ neutral molecules in a Coulomb crystal. A significant inverse kinetic isotope effect was observed, with $\mathrm{ND}_{3}$ reacting over three times faster than $\mathrm{NH}_{3}{ }^{106}$. This was explained in terms of the longer lifetime of the deuterated intermediate adduct formed en route to products. However, in this system, the experimentally measured rates for both isotopic species are lower than predicted by capture theory, indicating that once the collision complex is formed, it does not proceed with $100 \%$ probability towards products. The charge transfer process is relatively slow and dissociation of the complex back to reactants competes with the charge transfer reaction. Under these circumstances, the variation of the complex lifetime due to isotopic substitution, which is determined by the mass-dependent rate at which energy is transferred between vibrational states in the complex, becomes important to the overall reaction probability. Additional studies of ion-molecule reactions with small hydrogenated species are required to determine whether this is a general trend, or an observation specific to one particular reaction system.

Beyond the direct application to astrochemistry, the degree of quantum-state control achievable at low temperature offers us the potential to exert more control over the reaction pathways and outcomes, thereby enhancing our understanding of how reactions occur on a fundamental level. By combining an electostatic deflector with a linear Paul ion trap, the reactivity of different conformational isomers has been examined ${ }^{107}$. The $c i$ s and trans conformers of 3-aminophenol were spatially separated using an electrostatic deflector, as they have different dipole moments. The inhomogeneous electric field deflected the cis conformer to a greater extent, owing to its larger dipole moment. By preferentially directing the different conformers into the ion trap, the relative reactivity of each isomer with $\mathrm{Ca}^{+}$ions in a Coulomb crystal could be established ${ }^{107}$. Classical capture theory then helped to rationalize the differences in reactivity. The stronger ion-dipole interactions associated with cis-aminophenol results in a stronger long-range interaction with $\mathrm{Ca}^{+}$ions, yielding a larger rate coefficient compared to the trans conformer ${ }^{107}$. The same experimental set-up has been employed to separate para and ortho $\mathrm{H}_{2} \mathrm{O}$ molecules, enabling rotational-state-specific reaction rate coefficients to be measured for the proton transfer reaction $\mathrm{H}_{2} \mathrm{O}+\mathrm{N}_{2} \mathrm{H}^{+} \rightarrow \mathrm{N}_{2}+\mathrm{H}_{3} \mathrm{O}^{+}$ (see Fig. 1) ${ }^{108}$. A number of ion-molecule reactions have now been precisely studied in Coulomb crystals at collision energies from a few $\mathrm{K}$ up to a few hundred $\mathrm{K}^{31,98,109-120}$.

At lower collision energies, corresponding to temperatures down to a few $\mathrm{K}$, we start to observe more features characteristic of quantum behaviour. The inelastic collisions (those that lead to a change of quantum states of colliding molecules but not a change of chemical identity) of a number of neutral molecules have been precisely probed in a state-to-state manner using a crossed molecular beam set-up with a small crossing angle. Systems studied in this way include $\mathrm{CO}+\mathrm{H}_{2}{ }^{83}, \mathrm{O}_{2}+\mathrm{H}_{2}{ }^{84}$, $\mathrm{NO}+$ $\mathrm{He}^{85,89}$ and $\mathrm{NO}+\mathrm{H}_{2}{ }^{86}$ (in the latter two cases, with the properties of the NO reactant controlled by a Stark decelerator (Box 2)). In each of these systems, the experimental results were found to be well described by quantum scattering calculations, which could be performed at high accuracy owing to the relatively small size of the systems. In the case of $\mathrm{NO}+\mathrm{He}$, the measurements were performed over the energy range $0.2-8.5 \mathrm{~cm}^{-1}$ (an energy where very few partial waves contributed to the collision) and included imaging the angular distribution of the NO molecules scattered after the collision (the 'differential cross section'). At the energies of the fully resolved collisional resonances, the angular distributions showed strong differences between resonances, reflecting the underlying partial wave character of the collision that led to the resonance, and the very fine details of the potential energy surface. Quantum resonances have also been experimentally observed in the reactive scattering of $\mathrm{S}+\mathrm{H}_{2} \rightarrow \mathrm{SH}+\mathrm{H}$ and the equivalent $\mathrm{S}+\mathrm{HD}$ reactions at collision energies down to $5.8 \mathrm{~K}^{82,121}$. However, the experimental findings could not immediately be reconciled with theoretical predictions at low energies for S + HD system. In this case, a more thorough theoretical treatment is required, based on highly accurate singlet and triplet PESs and that accounts for non-adiabatic effects, to reproduce the experimentally-observed reaction dynamics of the system. Finally, the $\mathrm{F}+$ para- $\mathrm{H}_{2} \rightarrow$

$\mathrm{HF}+\mathrm{H}$ reaction (see above) was recently studied at low angles of intersection between the crossed reactant beams ${ }^{87}$. Quantum tunnelling through the barrier was found to be enhanced by a post-barrier resonance state, with the experimental findings supported by quantum scattering calculations ${ }^{87}$.

\section{From $1 \mathrm{~K}$ down to a few $\mathrm{mK}$}

As we move below the temperatures of the coldest naturally-occurring environments in the Universe, we start to see more frequent deviations from classical behaviour. With sub-K collision energies, we can probe the transition from classical to quantum mechanics in a range of different reaction systems, for example, by observing quantum phenomena such as scattering resonances. These become more prominent at low temperatures because the number of partial waves needed to describe the collision dynamics is small, hence reducing the 'smearing' of the resonances in the cross section. Under these conditions, reactions also become more sensitive to magnetic and electric fields, with these effects sometimes needing to be incorporated into quantum calculations. The effects of population of just one or two low- $\mathrm{J}$ rotational quantum states of the reactants also become more prominent.

A number of chemi-ionisation reactions have been studied at collision energies below $1 \mathrm{~K}$ using a merged beam approach (see Box 1). A detailed study of the relative reactivities of metastable $\mathrm{He}$ with $\mathrm{H}_{2}, \mathrm{HD}$ and $\mathrm{D}_{2}$ has revealed a quantum kinetic isotope effect, with scattering resonances occurring at different collision energies for each of the isotopologues of molecular 
hydrogen ${ }^{122}$. The role of rotational excitation has also been probed, with rotationally-excited ortho- $\mathrm{H}_{2}$ reacting faster than para- $\mathrm{H}_{2}$ in the ground state. The enhanced reactivity of ortho- $\mathrm{H}_{2}$ is attributed to the stronger long-range attraction in the intermolecular interaction potential resulting from the rotational excitation of the $\mathrm{H}_{2}$ reactant ${ }^{88,123}$. The influence of orientation effects has been explored in the reaction of $\mathrm{Ne}^{*}$ with $\mathrm{Ar}$ at collision energies down to $20 \mathrm{mK}$ using a merged beam apparatus, with the external magnetic field controlling the orientation of the electronic angular momentum of the $\mathrm{Ne}\left({ }^{3} \mathrm{P}_{2}\right)$ reactants ${ }^{124}$. Two chemi-ionisation reaction channels are present in this system: Penning ionisation that yields $\mathrm{Ne}\left({ }^{1} \mathrm{~S}\right)+\mathrm{Ar}+\mathrm{e}^{-}$products, and associative ionisation that results in $\mathrm{NeAr}^{+}+\mathrm{e}^{-}$. The product branching ratio exhibited a dependence on both the external magnetic field and the collision energy. At the lowest collision energies, $\mathrm{Ne}^{*}$ was found to reorient into the most favourable configuration for complex formation, preferentially forming the associative ionisation products. These findings highlight the ability of external fields to manipulate the outcome of a low-temperature collision process, in this case, by controlling the orientation of one of the reactants to favour a particular reaction channel.

An adapted version of the merged beam technique has been developed to facilitate the study of certain ion-molecule reactions, with a highly-excited Rydberg molecule employed in place of an ion. $\mathrm{H}_{2}^{*}$ Rydberg molecules can exhibit chemical reactivity that is analogous to that of $\mathrm{H}_{2}^{+}$ions. As the Rydberg electron shields the ionic core from stray fields, it is advantageous to use $\mathrm{H}_{2}^{*}$ in place of $\mathrm{H}_{2}^{+}$in a merged beam experiment ${ }^{125}$. At collision energies below $1 \mathrm{~K}$, the $\mathrm{H}_{2}^{*}+\mathrm{H}_{2} \rightarrow \mathrm{H}_{3}^{+}+\mathrm{H}+\mathrm{e}^{-}$ reaction proceeds with a higher rate coefficient than predicted by a classical Langevin capture model. This increase in the rate coefficient is attributed to the tendency for the reaction to follow the minimum energy pathway adiabatically. The $\mathrm{H}_{2}^{+}$ion core interacts with the rotational quadrupole moment of ortho- $\mathrm{H}_{2}(J=1)$, fixing the rotational axis of the $\mathrm{H}_{2}$ molecule to the collision axis and increasing the rate coefficient above what is predicted by Langevin theory ${ }^{126}$. This is another example in which a single low- $J$ rotational level of one of the reactants is populated, leading to behaviour that is distinct from that observed in thermal samples.

A number of ion-atom charge transfer reactions have been studied in hybrid trap set-ups at collision energies down to a few $\mathrm{mK}$, with many of these discussed in a recent review article ${ }^{60}$. By exciting the micromotion of the ionic reactant, it is possible to vary the energy of ion-atom collisions, thereby establishing the energy dependence of charge transfer processes at low and ultralow temperatures ${ }^{127}$. Over the past few years, hybrid traps have been used for more chemically complex systems, with the technique applied to the study of reactions between charged molecules and atoms. The reactive collisions of electronically excited $\mathrm{Ca}$ atoms with $\mathrm{BaCl}^{+}$ions have been probed at collision energies as low as $200 \mathrm{mK}$. The reaction rate for the $\mathrm{Ca}\left({ }^{1} \mathrm{P}\right)$ state is found to decrease with decreasing temperature in the range $0.3-2 \mathrm{~K}$, contrary to the predictions of capture theory, and the reaction is suppressed. In this case, the radiative lifetime of the excited $\mathrm{Ca}^{*}$ quantum state was found to play a key role in governing the observed reaction rate ${ }^{128}$. A theoretical model allows us to calculate the time necessary for the reactants to form the collision complex. The longer time necessary at lower temperatures to form a collision complex favours radiative decay over the formation of products. Charge transfer reactions have been examined in $\mathrm{Rb}+\mathrm{N}_{2}^{+}$and $\mathrm{Rb}+\mathrm{O}_{2}^{+}$at collision energies in the $\mathrm{mK}$ regime, with the interplay of short-range and long-range interactions giving rise to different charge transfer mechanisms in the different reaction pathways ${ }^{129}$. In systems where both reactant species are open-shell and spin-orbit effects may be important, there are multiple close lying potential energy surfaces that can potentially influence the reaction. A comparison between quasiclassical trajectory and quantum dynamics simulations in the $\mathrm{Rb}+\mathrm{N}_{2}^{+}$system indicated that the dynamics is dominated by non-adiabatic surface-hopping near the intersections of these surfaces. Reaction pathways proceeding through long-lived complexes also compete with direct collisional trajectories that lead to product formation. However, for these systems in which atoms heavier than $\mathrm{H}$ and $\mathrm{D}$ are transferred, tunneling effects are not important in the $\mathrm{mK}$ regime. The reactions of $\mathrm{Rb}$ with $\mathrm{N}_{2}^{+}$and $\mathrm{O}_{2}^{+}$also illustrate the competing factors that may influence the rate at which a reaction proceeds. It is not always obvious which parameter will play a dominant role in governing the reaction dynamics, therefore highly accurate theoretical approaches are needed to offer us a more complete understanding of the reaction process.

The vast majority of reactive collisions studied at energies between $1 \mathrm{mK}$ and $1 \mathrm{~K}$ involve either charged or electronically excited species as one of the reactants. Such species tend to be very reactive, even at low temperatures, as the reactions they participate in typically feature no energetic barriers. In general, such reactions produce a charged product, which can be detected with very high sensitivity. We are not aware of any experimental studies of reactive collisions between neutral, closed-shell molecular reactants at collision energies between $1 \mathrm{mK}$ and $1 \mathrm{~K}$, although the $\mathrm{H}$ atom exchange reaction between $\mathrm{Ca}$ and $\mathrm{LiH}$ has been studied at $\sim 1 \mathrm{~K}$ in cryogenic buffer gas cooled trap ${ }^{37}$. The next section highlights studies of reactive collisions of neutral diatomic alkali metal species in the ultracold below $1 \mathrm{mK}$. There have also been a number of studies involving non-reactive collisions between neutral species under cold conditions. Readers are directed to recent review articles for a detailed discussion of inelastic scattering under cold conditions ${ }^{131,132}$.

\section{Reactions in the ultracold regime below $1 \mathrm{mK}$}

With collision energies below $1 \mathrm{mK}$, almost all reaction parameters can be precisely controlled, allowing us to precisely establish the role each variable plays in influencing the reaction process. Very few partial waves, or even just a single partial 
wave, are involved in reactions at ultralow temperatures, substantially simplifying the reaction dynamics and bringing quantum effects to the fore. While ultralow collision temperatures can only be accessed for a small number of reactant species that can be formed and trapped at ultracold temperatures using the existing methods described in Box 5, there is still much we can learn about the forces and factors governing chemical reactivity in these systems. Reactions show increasing sensitivity to the fine structure, such as the small energy splittings of the PESs, and non-adiabaticity can become increasingly important, with the effects of geometric phase needing to be explicitly considered in some systems ${ }^{133}$. Geometric phase, which relates to the sign change that occurs in the electronic wavefunction when the nuclei of a reactive system circle a conical intersection between two PESs, has very rarely been observed in room temperature systems. This is because the effects of geometric phase are smeared out by the large number of partial waves contributing to the collision. However, in the $s$-wave or $p$-wave limit observed at ultracold temperatures, the effect is predicted by theory to be increasingly important and observable. For example, the inclusion or not of the geometric-phase effects results in a difference of up to an order of magnitude in the calculated state-to-state cross sections for the reaction $\mathrm{OH}+\mathrm{O} \rightarrow \mathrm{H}+\mathrm{O}_{2}{ }^{134}$ at temperatures below $1 \mathrm{mK}$. Quantum dynamics simulations at ultracold temperatures, which typically includes multiple close lying surfaces, become acutely sensitive to the accuracy of the computed PESs in part because of the prominence of such non-adiabatic processes.

The hybrid trapping technique has allowed ion-neutral systems to be studied at collision energies as low as $10 \mu \mathrm{K}$. The collisions of a single trapped ion with neutral ultracold buffer gas atoms have been monitored under conditions in which only a few partial collisional waves contribute. Ultracold Li atoms held in an optical trap were transferred to the centre of a Paul ion trap (Box 3), where they interacted with a single trapped $\mathrm{Yb}^{+}$ion ${ }^{135}$. The $\mathrm{Yb}^{+}$ion was sympathetically cooled by the Li atoms, with the collision energy of these interactions only $15 \%$ greater than the height of the $p$-wave centrifugal barrier (referred to in the original study as the $s$-wave limit, below which the reaction proceeds in a single collisional angular momentum state). The significant mass difference between $\mathrm{Yb}$ and $\mathrm{Li}$ was a crucial factor in reaching the quantum regime. Because of the strong mass imbalance, the $\mathrm{Yb}^{+}$ion only moved very slightly from the trap centre after colliding with a Li atom, minimising the heating that arises from micromotion in Paul traps when ions are not located in the trap centre. The interactions between atoms and ions arise from the electric dipole induced in the neutral atom as it approaches the ion, therefore the interaction potential exhibits $r^{-4}$ asymptotic behaviour and is dominated by long-range attractive forces. Although these long-range attractive forces make the centrifugal barrier heights very low, the low collision energy meant that only $s$-wave and $p$-wave interactions were energetically permitted (see Fig. 2). The probability of collision-induced spin exchange in the $\mathrm{Yb}^{+}$ion was found to display a strong dependence on the collision energy. This result provided strong evidence of quantum behaviour, which can be attributed to the effects of quantum reflection and quantisation of collisional angular momentum. In contrast, classical models predicted the probability of spin exchange to be independent of collision energy.

Using the state-of-the-art experimental methods described earlier in this Review, collisions have been studied in ensembles of gases at temperatures on the order of a few hundreds of $n K$ in systems including $\mathrm{KRb}^{137-139}, \mathrm{NaLi}^{140}$, triplet $\mathrm{Rb}_{2}{ }^{141}$, $\mathrm{Li}_{2}{ }^{142}, \mathrm{NaRb}^{143,144}, \mathrm{RbCs}^{81}, \mathrm{NaK}+\mathrm{K}^{145}$ and $\mathrm{NaK}^{146}$. For all these systems of trapped ultracold molecules, fast collisional trap-loss rates have been observed. Comparable loss rates have been reported for systems with reaction channels that are energetically feasible, such as $\mathrm{KRb}$, and for systems in which all reaction pathways are energetically forbidden, such as RbCs, $\mathrm{NaRb}$ and $\mathrm{NaK}^{81}$. Studies of non-reactive systems suggest that the formation of complexes, which are very long-lived at these temperatures, may play a key role in the observed trap loss. Recent experimental work on non-reactive ${ }^{87} \mathrm{Rb}^{133} \mathrm{Cs}$ molecules showed that two-body collision complexes can be optically excited by the trapping lasers, providing a possible mechanism to explain the high two-body loss rates ${ }^{147}$. This result has since been confirmed in an experimental study of reactive KRb molecules $^{148}$.

Exceptional progress has been made toward directly monitoring product formation in ultracold reactions. Explicitly detecting the products of ultracold bimolecular collisions adds another layer of technical complexity to what are already very challenging experiments. The products of exothermic reactions are generally more energetic than the reactants with a tendency to escape from the reaction volume, and cannot be easily cooled, trapped or detected under the experimental conditions. In spite of these technical challenges, all species involved in an ultracold bimolecular reaction have recently been directly observed (see Fig. 3). The reactants, reaction intermediates and products of the $\mathrm{KRb}+\mathrm{KRb} \rightarrow \mathrm{K}_{2} \mathrm{Rb}_{2}^{*} \rightarrow \mathrm{K}_{2}+\mathrm{Rb}_{2}$ system at a collisional temperature of $500 \mathrm{nK}$ have all been unambiguously identified through the complementary use of spectroscopic techniques and velocity-map-imaging methods ${ }^{43}$. This is the lowest temperature at which a bimolecular reaction has been observed to date. At such low temperatures, the number of accessible exit channels is severely limited, thereby significantly extending the lifetime of the reactive intermediate, which has been measured to be $360 \mathrm{~ns}^{148,149}$. The transient complex has been shown to live for orders of magnitude longer ( $\sim$ picoseconds) than one would expect from systems of a similar size at room temperature. The extended lifetime of the reaction complex also suggests that directly probing the properties of the reaction intermediate will be possible without requiring ultrafast lasers.

Recently, the $\mathrm{K}_{2}$ and $\mathrm{Rb}_{2}$ products of the bimolecular reaction between ultracold $\mathrm{KRb}$ molecules were state-selectively detected $^{150}$. It was observed that nuclear spin was preserved throughout the reaction process. Through the application of 
an external magnetic field, a variable superposition of the the nuclear spin states of the reactants could be populated and a corresponding change was observed in the distribution of the nuclear spin states of the products. The ability to manipulate and control the outcome of a reactive collision through the application of an external magnetic field beautifully demonstrates the power of combinations of ultracold techniques. This work also verifies 40 year old theoretical predictions of conservation of nuclear spin that leads to state-selected products ${ }^{151}$, which had previously only been observed in the reactions of very light hydrogen-containing species. Another recent study of spin conservation, relevant to ultracold collisions, shows that the rate coefficients for Penning ionisation between an $\mathrm{He}^{*}$ beam and electronically-excited ultracold Li could only be explained through conservation of electron spin and the projection of total molecular orbital angular momentum along the internuclear axis $(\Lambda)$ in the reaction ${ }^{152}$.

State-to-state chemical reactions have recently been experimentally demonstrated in an ultracold three-atom system, highlighting again how under these conditions it is possible to control a reaction at a quantum level. The recombination of three ultracold $\mathrm{Rb}$ atoms held in a dipole trap resulted in the formation of a weakly-bound $\mathrm{Rb}_{2}$ molecule, with the third atom stabilising the dimer by absorbing the excess energy from the exoergic association process. Because the majority of product dimers could be state-selectively detected, the influence of the initial states of the reactants on the resulting product state distribution could be experimentally established ${ }^{153}$.

A remarkable example of controlling stereochemistry was demonstrated ${ }^{154}$ by using an optical lattice trap to confine KRb polar molecules in a quasi-two-dimensional array — a pancake-like geometry — with the molecular dipoles oriented along the tight confinement direction. It was shown that the combination of sufficiently tight confinement and the Fermi statistics of the molecules resulted in the polar molecules approaching each other only in a 'side-by-side' collision under repulsive dipole-dipole interactions. The experiments revealed a two-order of magnitude suppression of the reaction rate coefficient under these conditions. This is clear evidence that the outcome of a chemical reaction can be manipulated through the use of external electric fields, trapping forces and ultracold conditions, which allow precise control of the relative orientation of colliding dipoles.

Accompanying these experimental advances, increasingly accurate theoretical work has been undertaken to provide benchmark calculations for the anticipated molecular reaction dynamics. Following the construction of an accurate ab initio PES, ultracold reactive collisions of the heavy alkali system $\mathrm{K}+\mathrm{KRb} \rightarrow \mathrm{K}_{2}+\mathrm{Rb}$ have been theoretically studied using numerically exact quantum dynamics methods ${ }^{155}$. Trajectory calculations found that long-range interactions played a key role in dictating the reaction rate coefficient, as one might intuitively expect from capture theory. The predicted rotationally-resolved reaction rates were statistical, with the rotational states well described by a Poisson distribution. This behaviour was attributed to the chaotic nature of the energy states of the short-range three-heavy-atom collision complex formed during the reaction process. The $\mathrm{K}_{2} \mathrm{Rb}$ reaction intermediate is postulated to play a crucial role in determining the outcome of the collision ${ }^{155}$.

Extreme control over ultracold bimolecular collisions has been exercised in a very recent study involving $\mathrm{CaF}+\mathrm{CaF}$ collisions. By transferring laser-cooled ${ }^{40} \mathrm{Ca}^{19} \mathrm{~F}$ molecules from a magneto-optical trap (MOT) into an optical dipole trap and finally into an array of optical tweezers, it has been possible to manipulate the quantum states and control the collisions of individual pairs of molecules. This enabled the study of collisions between pairs of CaF molecules with unprecedented precision $^{58,156}$. Two optical tweezer traps, each featuring a single state-selected CaF molecule (including selection of hyperfine states), were merged. After a selected time, the combined optical tweezer trap was separated again, with any remaining CaF molecules state-selectively detected. This set-up is almost ideal for the study of two-body interactions because it allows the manipulation of single molecules. Two-body collisional loss rates were extracted for CaF molecules in their ground ro-vibrational state and for selected excited hyperfine states. The formation of long-lived reaction intermediates and possible chemical reactions ( such as, $\mathrm{CaF}+\mathrm{CaF} \rightarrow \mathrm{CaF}_{2}+\mathrm{Ca}$ ) were proposed as the likely mechanisms responsible for the observed collisional trap loss rates ${ }^{156}$. If product detection can be added to the extreme control that the technique provides over the properties of the reactants, we anticipate that the use of optical tweezers will offer new chemical insights into reactive collisions in the years to come.

\section{Conclusion and future directions}

This Review has highlighted some of the fascinating recent studies of chemical reactivity at temperatures approaching absolute zero. Extreme and artificially imposed ultracold conditions are already offering new insights into how molecular processes occur under such conditions, showing effects that are not observed at higher temperatures. We have discussed the complicated temperature dependence of reaction rates in the cold regime down to $1 \mathrm{mK}$, and the effects of the very long-lived complexes and the resonant energy states of such complexes on the reaction outcomes at both cold and ultracold temperatures. We have also considered the possibility of controlling stereochemistry and reaction outcomes using electric and magnetic fields, including the deployment of optical-trap 2D arrays. Furthermore, we have discussed the transition from the classical to the quantum regime observed at very low temperatures and effects such as quantum tunnelling and barrier reflection, geometric phase and other non-adiabatic effects that become more prominent as the temperature is lowered, occurring under the conditions of single 
or few partial collisional waves, and with only the lowest rotational quantum states populated.

Despite the sustained efforts of many researchers over more than 20 years, the field of ultracold chemistry is still very much in its infancy. Much of the work in this field has been focused on developing techniques to produce and control molecular samples at very low temperatures. These primary developments were needed to generate sufficiently dense ensembles of cold molecules and to develop methodologies to characterise and monitor these samples, before reaction studies could be conducted. The study of chemical reactions under cold and ultracold conditions offers exciting opportunities to understand, for example, chemistry in the remote areas of our Universe, as well as to characterise the quantum effects on reactivity highlighted above, but it also presents an impressive set of experimental challenges. Creating and combining cold samples of two or more chemical species, overlapping in time and space, is technically complex. Also, because most cold-collision and ultracold-collision experiments involve low density samples, the probability of collisions occurring at all in the measurement timescale can be very low, hence the density of product species is very low. Finally, the detection of products and their quantum states is generally much more difficult than detecting the reactants. Whereas reactant species are typically prepared in a few selected quantum states, or a single state, and can be detected by exciting a small number of spectroscopic transitions, the product species may be formed in states that are widely dispersed in energy. This is especially true for strongly exothermic reactions, for which spectroscopic detection methods require almost single molecule sensitivity to be effective ${ }^{150}$.

In this Review, we have presented experiments and results that cover more than eight orders of magnitude on the temperature scale (from 10s of $\mathrm{K}$ down to $100 \mathrm{~s}$ of $\mathrm{nK}$ ). However, as pointed out earlier, there is no single chemical reaction to date that has been studied experimentally over that full temperature range, as most studies focused on only one or two orders of magnitude. This is due to the limited temperature range over which the techniques to generate cold or ultracold species can be applied and the tendency for any experimental setup to be tailored to a narrow range of chemical species. For example, the optical traps described here are relatively shallow, and hence can only be used in the ultracold regime. As a result, some of the most intriguing questions about the transition from the classical to the quantum regime are difficult to answer at this point in time. In practice, the transition occurs over many orders of magnitude on the temperature (or energy) scale. Quantum effects do not suddenly switch off at higher temperatures, they simply become less prominent in the experimental observations relative to classical effects. This is a consequence of the thermal averaging that occurs at higher temperatures and of the decreasing deBroglie wavelength of the particles as the temperature increases. The extent to which quantum effects - that are known to be prominent at ultracold temperatures - should be considered over the higher range of temperatures that chemical reactions occur in ambient laboratory conditions or in natural environments is still unclear. The only way to fully answer this question is to make a complete and continuous link, by traversing the ultracold and cold regimes all the way up to room temperature, in experimental measurements and theory work. Ideally, such studies would consider different classes of chemical reactions, including a comparison between ion-neutral and neutral-neutral reactions. Such studies would enable us to examine, for example, the broad collisional resonances associated with the short lifetimes of collision complexes, the onset of the deviation from classical capture predictions owing to quantum reflection and tunnelling, and the transition in behaviour that occurs when moving from single partial-wave collisions, to a few partial waves, to multiple partial waves. The best possible test for the accuracy of calculated reaction PESs is to measure reaction rate coefficients (and other properties, such as chemical product branching ratios, vibrational product quantum states and complex lifetimes) over wide collision energy ranges, because different parts of the PESs will be sampled at higher energies.

Although there is much interest in the new aspects of chemical reactivity that have been observed for some model reaction systems, many of the chemical species that have been studied under ultracold conditions (including the dimers of alkali metal atoms) might not normally be considered to be of broader chemical interest. 'Real' chemical reactions typically have a high level of complexity, involving polyatomic species with many sites of potential reactivity, complex reaction pathways and multiple product channels. We have seen in this Review that chemical reaction systems exhibit a different physical behaviour at very low temperatures, but does this lead to different chemical outcomes, including the production of exotic product molecules, or very different branching ratios for the possible product-molecule combinations? Is there really 'new chemistry' at such low temperatures? A key motivation for chemists in exploring the ultracold regime is to see whether these detailed processes, often with outcomes (for example, reaction yields) that are hard to predict theoretically before experimental measurements are made, can be tamed through exercising extreme control over the reaction conditions, collision properties and quantum states. This goal is likely to remain a challenge for many years to come. Attaining it will require more general methods for making cold molecular samples, including chemically complex polyatomic species - methods that can achieve temperatures orders of magnitude lower than currently attainable by collisional cooling with cold buffer gases. Some recent progress has been made towards this goal. Sub-K temperatures have been reached in a system that is not amenable to laser cooling, with the co-trapping of Zeeman-decelerated $\mathrm{C}$ atoms and $\mathrm{O}_{2}$ molecules. This work paves the way for studying the astrochemically-relevant $\mathrm{C}+\mathrm{O}_{2}$ $\rightarrow \mathrm{CO}+\mathrm{O}$ reaction under cold conditions, a system that has previously been studied only at temperatures spanning $10 \mathrm{~s}$ to $100 \mathrm{~s}$ of $\mathrm{K}^{157}$.

In the early years of the study of chemistry at very low temperatures, the direct laser cooling of molecules was considered 
to be so challenging to be unachievable for more than a few diatomic species with very favourable properties. The direct laser cooling of molecules to temperatures where they could be trapped (sub-mK) was considered by many to be a mere dream. The complex energy level structure of polyatomic species, and the tendency for excited states (populated by the laser cooling process) to decay to large numbers of lower states, thus breaking the closed optical cycle cooling process, is one of the principal limitations. Heroic efforts over the past decade have demonstrated that laser cooling to sub-mK temperatures is indeed possible for selected diatomic molecular species. Progress is being made with techniques that efficiently pump population out of lower states and back to the upper state where it came from, effectively 'closing' the cooling cycle. Although this approach involves multiple lasers, such lasers can be inexpensively produced at single frequencies ${ }^{66}$. The extension of this progress to a wide range of chemical species, with even some polyatomic molecules successfully laser cooled ${ }^{64,68}$, perhaps offers the greatest opportunity to study complex chemical processes over many orders of magnitude on the temperature scale.

In conclusion, the examples illustrated in this Review - including the observation of collision resonance effects in reactions at high resolution using merged beams, the recent demonstrations of collisions between single laser-cooled CaF molecules trapped by optical tweezers, the control of orientation of dipoles in two-dimensional optical trap arrays, and the characterisation of reaction intermediate lifetimes at ultracold temperatures in the $\mathrm{KRb}+\mathrm{KRb}$ reactions - are remarkable demonstrations of the richness of current studies and those that are yet to come. The possibility of observing chemical reactions with quantum-degenerate molecular samples is likely to add new perspectives on many-body effects in the future. In parallel, the extension of studies to wider chemical reaction types and spanning the transition from the cold to the ultracold will bring important new general insights into chemical reactivity and the ability to control chemical processes.

\section{Acknowledgements}

We are grateful for financial support from the Engineering and Physical Sciences Research Council (B.R.H. and T.P.S.) and the Royal Society (B.R.H.), and for the careful reading of the reviewers of this manuscript.

\section{Author contributions}

Both authors contributed equally to all aspects of the article.

\section{Competing interests}

The authors declare no competing interests.

\section{Peer-reviews information}

Nature Reviews Chemistry thanks the anonymous reviewers for their contribution to the peer review of this work.

\section{Publisher's note}

Springer Nature remains neutral with regard to jurisdictional claims in published maps and institutional affiliations.

\section{Editor Summary}

Chemical reactions can still occur at temperatures as low as $1 \mu \mathrm{K}$. Under such conditions, however, quantum effects are increasingly important, challenging the common understanding of chemical reactivity. The current developments of experimental and theoretical approaches are starting to provide us with a clearer picture of chemistry close to absolute zero. 


\section{Box 1: Cold chemistry using the relative velocity of supersonic beams}

The study of reactions by the crossing of two supersonic beams, each containing one of the reactants, was pioneered by Herschbach, Lee and co-workers and recognised in the 1986 Chemistry Nobel Prize citation as having given rise to the field of reaction dynamics ${ }^{6}$. The ability to minimise and control the internal energy of the reactants in supersonic beams revolutionised our understanding of how elementary chemical reactions take place. It was recognised, however, that the high relative velocity of reactants in beams crossed at $90^{\circ}$ gives rise to a significant collision energy and, hence, high effective collisional temperatures. Several decades later, experiments have reached lower collision energies by adopting lower crossing angles with beams of comparable velocity, at the cost of greater experimental complexity. Very recently, a crossing angle of $4^{\circ}$ (corresponding to collision energies around $1 \mathrm{~K}$ ) was achieved using a dual-beam-valve set-up, in which the two pulsed valves are placed side by side outside the vacuum chamber. The beams generated by each valve are injected into the same vacuum chamber with a small initial separation ${ }^{7}$.

Collision energies lower than $1 \mathrm{~K}$ have been reached by merging supersonic beams onto a single axis, which is equivalent to crossing beams at an angle of $0^{\circ}$. If only one of the beams contains paramagnetic reactants, that beam can be directed by a magnetic guide onto the axis of a second (non-paramagnetic) beam ${ }^{8}$. The figure shows the schematic of a merged beam experiment in which two reactants, either $\mathrm{H}_{2}$ or Ar reacting with metastable $\mathrm{He}$ atoms, are supersonically expanded and skimmed as separate beams. A curved magnetic quadrupole-guide (inset) bends the beam of metastable He atoms onto the axis of the undeflected $\mathrm{H}_{2}$ or Ar reactants, yielding a single merged beam (purple). Alternatively, both reactant beams can be guided. In this case, a magnetic guide can direct a beam of paramagnetic reactants and an electrostatic guide can bend a beam of polar reactants onto a common axis 9 .

The collision energy in a merged beam experiment is dependent on the relative velocity of the two beams along the merged axis, and the velocity spread within each beam. A careful match of the beam velocities, the use of ultra-short gas pulses ${ }^{10}$, and measurements on a small portion of the merged gas pulses (taking advantage of the correlation between velocity and position in the gas pulse) has enabled chemi-ionisation reactions to be studied at collision energies as low as $10 \mathrm{mK}^{11}$.

It is also possible to study reactions between molecular reactants entrained in a single supersonic expansion. An apparatus based on reaction kinetics in uniform supersonic flow (CRESU, cinétique de réaction en ecoulement supersonique uniforme) has been used to study a number of reactions of interstellar relevance over the past several decades, reaching collision energies as low as $5.8 \mathrm{~K}^{12,13}$. Central to the CRESU approach is the use of a Laval nozzle, in which the combination of convergent and divergent sections results in a uniform and thermally-equilibrated beam. Reactions are typically initiated by producing one of the reactant species (for example, by laser photolysis) in the beam shortly after the nozzle.

Another method for studying intra-beam collisions takes advantage of the different velocities — the velocity slip — naturally exhibited by different species in a single supersonic beam. This velocity slip can be large or small, depending on the difference of the masses of the two reactants and on the experimental conditions employed (for example the pressure of the gases behind the pulsed-beam valve). Narrow velocity distributions can be achieved by using short gas pulses and long flight times ${ }^{14-16}$.

Figure adapted with permission from Ref. ${ }^{8}$

\section{Box 2: Decelerators and traps to reduce the absolute reactant velocities}

An alternative approach to the use of merged beams (Box 1) is to lower the collision energy using electrical, magnetic, or optical fields, or mechanical methods, to reduce the absolute forward velocity of a subset of particles within a beam. The most widely adopted methods exploit the Stark effect, whereby an inhomogeneous, time-varying electric field is applied to a series of electrodes to decelerate polar species by a small amount at each stage ${ }^{17}$. A similar approach exploits the Zeeman effect to decelerate paramagnetic species, in which inhomogeneous and time-varying magnetic fields are applied to a series of electromagnets ${ }^{19,20}$. Stark and Zeeman decelerators have succeeded in generating packets of particles in selected quantum states with a narrow, well-defined and tunable forward-velocity distribution ${ }^{21,22}$.

Early efforts in the use of Stark decelerators attempted to study collisions by crossing two decelerated beams ${ }^{18}$. However, the combination of two very low-density reactant beams led to extremely low product yields, making product detection unachievable. The majority of collisional studies involving decelerated beams have combined decelerators with non-decelerated beams or with trapped samples for the study of inelastic collisions. The low density of decelerated reactants makes the detection of reactive collisions challenging, with product count rates as low as two molecules per second under typical operating conditions ${ }^{23}$

The deceleration of species to very low velocities opens the possibility of confining them in traps using electromagnetic or optical fields, thus increasing the timescale and sensitivity of experimental measurements. Because traps for neutral species are shallow compared to those for ionic species, neutral species can typically only be loaded into a trap if they are first translationally cooled (for example, by a decelerator or buffer gas cooling methods). Electrostatic traps can be employed to confine cold polar molecules, whereas electromagnetic, permanent magnetic or superconducting magnetic traps can be implemented to confine paramagnetic species ${ }^{24-29}$. 
For the study of low-temperature reactions with ensembles of trapped species, the reacting species can be co-trapped, with the time evolution of the reactant or product densities monitored for as long as the gases are trapped ${ }^{37,69}$. Alternatively, one species can be trapped while the other is passed through the trap, for example, in the form of a decelerated or velocity-filtered beam. The challenge of the co-trapping approach is that the trapping of species and the effective temperature attainable typically depend on molecular properties (such as the electric or magnetic moment, or polarisability) or optical properties. Therefore, the design of traps often needs to be tailored to one particular species, making the trapping of two species difficult. This can be circumvented by superimposing two traps, but such an approach is complicated because one must avoid interference between the trapping fields and achieve a matched trapping volume for the two species.

\section{Box 3: Studying ion-neutral reactions using trapped ions}

Methods for producing and trapping cold samples of ionic species (predominantly applied to cations) are very different from those adopted to study neutral species. While there is a range of ion trap designs available, linear radio-frequency (RF) ion traps are the most widely adopted for cold chemistry applications. One of the most frequently implemented types of RF trap, a linear Paul ion trap, makes use of a combination of oscillating and static electric fields applied to the trap electrodes, dynamically confining charged species to the trap centre. As outlined in Box 2 for the case of neutral traps, ion traps can be combined with other techniques (in particular, with sources of cold neutral molecules) for the study of ion-molecule reactions under cold conditions. For example, an experimental set-up for the study of ion-radical reactions is shown below (part a), which consists of a 12-stage Zeeman decelerator (see Box 2) combined with a magnetic guide and ion trap ${ }^{56}$. The simulated trajectories of particles as they pass through the magnetic guide region are also shown (part b). Target radical species are deflected by the Halbach arrays (that is, permanent magnets in a hexapolar configuration) around two skimming blades, enabling these species to reach the ion-trap region. All other species, such as undecelerated radicals and the seed gas, are physically blocked.

One of the key advantages of RF ion traps is their considerable trap depth, typically on the order of $1-10 \mathrm{eV}^{30}$. This allows for extended reactant trapping lifetimes, with the possibility to trap also the ionic products of exoergic reactive collisions. This provides an unambiguous identification of reaction products and a means of measuring branching ratios in complex systems with competing reaction channels.

To reach temperatures down to the mK regime, certain kinds of atomic ions held within a linear Paul trap (typically alkaline earth atomic ions) can be laser cooled. Under these conditions Coulomb crystals may be formed. These are regular three dimensional arrays of ions that can be maintained for long periods (typically hours) under ultrahigh vacuum conditions because of the depth of ionic traps. The positions of the laser-cooled ions within the Coulomb crystal can be directly observed, with fluorescence emitted as part of the laser cooling cycle collected by a microscope and camera imaging system (part c). The progress of the reaction can frequently be observed in real time by monitoring the change in the position of the laser-cooled ions within the crystal as a function of time. All ions within the crystal can be ejected through a flight tube and onto a microchannel plate detector at a selected reaction time, providing an additional method for the measurement of reaction rate coefficients and branching ratios 57 .

Other trapped ions (for example, molecular ions) may be sympathetically cooled and co-crystallised through their interaction with the laser-cooled species in the Coulomb crystals. In this way, the reactions of sympathetically cooled molecular ions can be studied under cold temperatures, in addition to the reactions of the laser-cooled atomic ions ${ }^{31}$. It is also possible to trap single ions or pairs of ions (for example, one laser-cooled ion and one sympathetically-cooled ion) at very low temperatures (sub $\mathrm{mK}$ ), achieving single particle control for chemical reaction studies. Higher order traps, particularly the 22-pole trap, have been used for the study of ion-neutral reactions over a wide temperature range (10-300 K). Instead of laser cooling, which is challenging to implement in a higher-order multipole trap as the ions are not as tightly confined to the trap centre, trapped ions can be collisionally cooled using a cryogenic buffer gas (such as $\mathrm{Ne}$ or $\mathrm{He})^{30}$.

Parts $\mathrm{a}$ and $\mathrm{b}$ are adapted with permission from $\operatorname{Ref}^{56}$. Part $\mathrm{c}$ is adapted with permission from Ref. ${ }^{57}$

\section{Box 4: Alternative methods for studying cold chemistry over the range $10 \mathrm{~K}-1 \mathrm{~K}$}

Complementary methods for producing or manipulating cold particles have been combined in innovative ways, to exert as much control as possible over the properties of the reactants. A number of these combinations are noted in Boxes 1-3. In addition to the combination of beam-based and trap-based methods, a number of alternative approaches have also been developed. These methods include electrostatic-guide velocity filters ${ }^{33}$, a centrifuge decelerator ${ }^{34}$, and Sisyphus cooling methods ${ }^{35}$. All of these techniques target small polar polyatomic molecules such as $\mathrm{CH}_{3} \mathrm{~F}, \mathrm{NH}_{3}$ and $\mathrm{H}_{2} \mathrm{O}$.

Reactions have been studied at temperatures of 1-10 K in buffer gas cells, where a cold buffer gas (usually helium at a temperature of a few K) collisionally cools both the internal and external degrees of freedom of the species of interest ${ }^{36,37}$. Buffer gas cooling methods are widely applicable ${ }^{38}$ and have also been effectively combined with traps and with velocity filters ${ }^{42}$.

Reactions can also be studied at temperatures $\sim 1 \mathrm{~K}$ within He nanodroplets. Species incorporated into He nanodroplets are cooled by elastic and inelastic collisions, with the energy dissipated by the evaporation of He atoms from the droplet ${ }^{39}$. As the 
He nanodroplets are typically chemically inert and spectroscopically transparent, they provide a useful means for studying clusters and reaction complexes at low temperatures, although the He nanodroplet 'solvent' may have some impact on the reaction rate $^{40,41}$.

\section{Box 5: Ultracold chemistry in traps with laser-cooling methods}

The combination of laser cooling with optical or magneto-optical trapping (MOT) lies at the heart of methods that enable chemical reactivity to be examined in the ultracold regime, with bimolecular reactions having been studied at temperatures as low as $500 \mathrm{nK}^{43}$. In principle, there are two approaches that may be deployed: direct laser cooling and trapping of molecules, or laser cooling of atoms followed by association of the atoms to form molecules. The latter has been more frequently implemented, but has been almost exclusively limited to species created by asssociating laser-coooled alkali metal atoms ${ }^{44-50}$. The methodology for the former approach is developing rapidly and has been applied for a small, but growing, number of molecular species to date ${ }^{66}$.

As first demonstrated over four decades ago, certain trapped atomic species are amenable to laser cooling, as long as a closed cooling cycle is optically accessible, and the excited electronic energy levels exhibit favourable lifetimes and transition probabilities $^{61}$. By combining laser cooling with evaporative cooling, temperatures in the range of a few hundreds of nK are routinely achievable for alkali metal neutral atom systems.

MOTs combine counter-propagating lasers with a magnetic quadrupole field to cool and spatially confine neutral atoms ${ }^{51}$, with the technique recently extended to selected molecular species ${ }^{52,53}$. Laser cooled atoms (for example, Rb) can be combined to form ultracold molecules (such as $\mathrm{Rb}_{2}$ ) using photoassociation or magnetoassociation methods ${ }^{54,55}$. Bringing atoms together in this way generally produces molecules with high internal energy and, therefore, coherent simulated emission pumping methods such as stimulated Raman adiabatic passage (STIRAP) are commonly adopted to move the population to the ground state $^{46}$. The molecules formed by magnetoassociation are generally not paramagnetic in the same way that their constituent atoms are and, hence, are not trapped by the MOT that is used for confining the laser cooled atoms. Optical dipole trapping is commonly used to achieve confinement of the ultracold molecules. Subsequent reaction studies can either involve collisions between pairs of ultracold molecules or between the laser cooled atoms and the molecular species.

Optical dipole traps rely on the electric dipole interaction of the neutral species with a focused laser. As optical forces are relatively weak, optical traps are shallow compared to the traps described in Boxes 2 and 3, achieving trap depths around 1 mK. Neutral species can be manipulated by optical tweezer traps ${ }^{58}$, with the AC Stark effect (Box 2) typically attracting atoms to the point of highest intensity in a tightly focused laser beam. To increase the density of the gas, the species of interest may be first trapped in an optical dipole trap before being transferred into the tweezer traps.

Researchers have combined traps for ions and atoms to study ion-neutral collisions over a temperature range that extends from 10 s of $\mathrm{K}$ down to $10 \mathrm{~s}$ of $\mu \mathrm{K}$. These hybrid ion-atom traps spatially and temporally overlap ensembles of trapped ions with ultracold trapped atoms. Hybrid trapping experiments were first reported in 2009, realised through the combination of a linear Paul trap with a MOT ${ }^{59,60}$

The presence of additional vibrational and rotational energy levels in molecules makes their laser cooling far more complex than that of atoms. Aided by particularly favourable Franck-Condon factors and a sufficient number of lasers to repump the population out of 'shelf states' (for example, states that are not part of the main laser cooling cycle), laser cooling has now been successfully used for a small number of molecules including $\mathrm{SrF}, \mathrm{CaF}, \mathrm{YbF}, \mathrm{YO}, \mathrm{SrOH}$ and $\mathrm{CaOCH}_{3}{ }^{62-68}$. The laser cooling of an increasingly diverse range of molecular species opens up the prospect of studying a more diverse range of chemical reactions at low temperatures in the future.

Evaporative cooling is an attractive prospect for preparing trapped ensembles of cold molecules that are not amenable to laser cooling and also for increasing the phase space density of molecules that have been laser cooled. It relies on the principle that the hottest species in an ensemble will evaporate from a trap, with the remaining species undergoing elastic collisions and rethermalising to reach lower temperatures. For efficient evaporative cooling to occur, there must be far more elastic collisions (where only kinetic energy is exchanged) than inelastic (state-changing) collisions between co-trapped species. Evaporative cooling has been theoretically and experimentally explored in a number of systems at temperatures below $1 \mathrm{~K}$ (see, for example, references 24,25,69-72). One of the ongoing challenges with achieving efficient evaporative cooling is the great number of parameters that must be considered and optimised to enhance the rate of elastic (favourable) to inelastic (unfavorable) collisions. For example, external fields can influence the rate at which elastic and inelastic collisions occur ${ }^{70}$. There is a delicate balance between having a sufficient number of cold molecules to perform the measurements of interest, without increasing the density to a point that can cause substantial trap losses as a result of increased inelastic collisions ${ }^{25}$. 
Figure 1. Exploring the reactivity of different isomers in ion-molecule reactions. A pulsed molecular beam of water is directed through two skimmers into an electrostatic deflector, where the inhomogeneous electric fields spatially separate para $-\mathrm{H}_{2} \mathrm{O}$ and ortho $-\mathrm{H}_{2} \mathrm{O}$. The strength of the electric field in the $x-y$ plane is shown in the inset plot. One component of the beam is preferentially directed into an ion trap containing a bi-component Coulomb crystal, where the $\mathrm{H}_{2} \mathrm{O}$ reacts with sympathetically cooled $\mathrm{N}_{2} \mathrm{H}^{+}$ions. Crystals can be ejected from the trap and onto a microchannel plate (MCP) detector for the recording of time-of-flight mass spectra (TOF-MS). Reproduced from Ref. ${ }^{108}$ under the terms of the Creative Commons CC BY license.

Figure 2. A hybrid trap for ions and neutral atoms.a. The graph illustrates the energy, $E$, of the atom-ion interaction potential as a function of the internuclear separation, $R$, in a hybrid ion-neutral trap for the lowest few partial waves. Operating at a collision energy indicated by the dashed black line, the only possible atom-ion pair close encounters are those involving $s$-wave and $p$-wave potentials. As the collision energy is very low, energetic barriers prevent $d$ and $f$ partial waves from contributing. Inset shows a schematic representations of a single heavy ion colliding with lighter neutral atoms in a hybrid ion-neutral trap. b. A cloud of ultracold ${ }^{6} \mathrm{Li}$ atoms are prepared in an optical trap. The atom cloud is moved into the centre of the Paul ion trap (where a single ${ }^{171} \mathrm{Yb}^{+}$ion is located) using piezo-controlled mirrors. The purple and red beams are from the cooling and trapping lasers, respectively, with $\lambda / 2$ indicating a half-wave plate. Part a is adapted with permission from Ref. ${ }^{136}$. Part $b$ is adapted with permission from ref. ${ }^{135}$

Figure 3. An ultracold bimolecular reaction study. The relative energies of the reactants, intermediates and products involved in the ultracold reaction $\mathrm{KRb}+\mathrm{KRb} \rightarrow \mathrm{K}_{2} \mathrm{Rb}_{2}^{*} \rightarrow \mathrm{K}_{2}+\mathrm{Rb}_{2}$ are shown. The energies are established from a combination of spectroscopic data and calculations. Two energetically forbidden channels are also illustrated. The channel described as the 'tetratomic reaction pathway' is exothermic and does not exhibit any energetic barriers to reaction, and is therefore energetically open. The density of states at the transient intermediate complex, $\mathrm{K}_{2} \mathrm{Rb}_{2}^{*}$, at the incident energy is denoted as $\rho_{c}(E)$. There are $N_{0}$ exit channels that can be populated at the incident energy. The inset plot illustrates the experimentally measured decay of $\mathrm{KRb}$ reactant molecules as a function of time, as established by ionisation detection (see reference 43 for more details). Adapted with permission from Ref. ${ }^{43}$ 


\section{References}

1. S. Arrhenius. Über die Reaktionsgeschwindigkeit bei der Inversion von Rohrzucker durch Säuren. Z. Physik. Chem. 4, 226-248 (1889).

2. B. A. McGuire. 2018 Census of Interstellar, Circumstellar, Extragalactic, Protoplanetary Disk, and Exoplanetary Molecules. Astrophys. J. Suppl. Ser. 239, 17 (2018).

3. The Nobel Prize in Physics, 1997. https://www.nobelprize.org/prizes/physics/1997/summary/

4. J. L. Bohn, A. M. Rey and J. Ye. Cold molecules: Progress in quantum engineering of chemistry and quantum matter. Science 357, 1002-1010 (2017).

5. J. C. Mather, D. J. Fixsen, R. A. Shafer, C. Mosier and D. T. Wilkinson. Calibrator Design for the COBE Far-Infrared Absolute Spectrophotometer (FIRAS). Astrophys. J. 512, 511-520 (1999).

6. The Nobel Prize in Chemistry, 1986. https://www.nobelprize.org/prizes/chemistry/1986/summary/

7. C. Amarasinghe, H. Li, C. A. Perera, M. Besemer, J. Zuo, C. Xie, A. van der Avoird, G. C. Groenenboom, H. Guo, J. Kłos and A. G. Suits. State-to-state scattering of highly vibrationally excited NO at broadly tunable energies. Nat. Chem. doi.org/10.1038/s41557-020-0466-8 (2020).

8. A. B. Henson, S. Gersten, Y. Shagam, J. Narevicius and E. Narevicius. Observation of Resonances in Penning Ionization Reactions at Sub-Kelvin Temperatures in Merged Beams. Science 338, 234-238 (2012).

9. J. Jankunas, B. Bertsche, K. Jachymski, M. Hapka and A. Osterwalder. Dynamics of gas phase $\mathrm{Ne}^{*}+\mathrm{NH}_{3}$ and $\mathrm{Ne}^{*}+\mathrm{ND}_{3}$ Penning ionisation at low temperatures. J. Chem. Phys. 140, 244302 (2014).

10. U. Even. The Even-Lavie valve as a source for high intensity supersonic beam. Eur. Phys. J. Techn. Instrum. 2, 17 (2015).

11. Y. Shagam and E. Narevicius. Sub-Kelvin Collision Temperatures in Merged Neutral Beams by Correlation in Phase-Space. J. Phys. Chem. C 117, 22454-22461 (2013).

12. B. R. Rowe, G. Dupeyrat, J. B. Marquette, D. Smith, N. G. Adams and E. E. Ferguson. The reaction $\mathrm{O}_{2}^{+}+\mathrm{CH}_{4} \rightarrow \mathrm{CH}_{3} \mathrm{O}_{2}^{+}$ + H studied from 20 to $560 \mathrm{~K}$ in a supersonic jet and in a SIFT. J. Chem. Phys. 80, 241--245 (1984).

13. C. Berteloite, M. Lara, A. Bergeat, S. D. Le Picard, F. Dayou, K. M. Hickson, A. Canosa, C. Naulin, J.-M. Launay, I. R. Sims and M. Costes. Kinetics and Dynamics of the $\mathrm{S}\left({ }^{1} \mathrm{D}_{2}\right)+\mathrm{H}_{2} \rightarrow \mathrm{SH}+\mathrm{H}$ Reaction at Very Low Temperatures and Collision Energies. Phys. Rev. Lett. 105, 203201 (2010).

14. C. Amarasinghe and A. G. Suits. Intrabeam Scattering for Ultracold Collisions. J. Phys. Chem. Lett. 8, 5153-5159 (2017).

15. W. E. Perreault, N. Mukherjee and R. N. Zare. $\operatorname{HD}(v=1, j=2, m)$ orientation controls HD-He rotationally inelastic scattering near 1 K. J. Chem. Phys. 150, 174301 (2019).

16. K. Gawlas and S. D. Hogan. Rydberg-State-Resolved Resonant Energy Transfer in Cold Electric-Field-Controlled Intrabeam Collisions of $\mathrm{NH}_{3}$ with Rydberg He Atoms. J. Phys. Chem. Lett. 11, $83-87$ (2020).

17. H. L. Bethlem, G. Berden and G. Meijer. Decelerating Neutral Dipolar Molecules. Phys. Rev. Lett. 83, 1558 (1999).

18. S. Y. T. van de Meerakker and G. Meijer. Collision experiments with Stark decelerated beams. Faraday Discuss. 142 113-126 (2009)

19. N. Vanhaecke, U. Meier, M. Andrist, B. H. Meier and F. Merkt. Multistage Zeeman deceleration of hydrogen atoms. Phys. Rev. A 75, 031402(R) (2007).

20. E. Narevicius, A. Libson, C. G. Parthey, I. Chavez, J. Narevicius, U. Even and M. G. Raizen. Stopping Supersonic Beams with a Series of Pulsed Electromagnetic Coils: An Atomic Coilgun. Phys. Rev. Lett. 100, 093003 (2008).

21. S. D. Hogan, M. Motsch and F. Merkt. Deceleration of supersonic beams using inhomogeneous electric and magnetic fields. Phys. Chem. Chem. Phys. 13, 18705-18723 (2011).

22. S. Y. T. van de Meerakker, H. L. Bethlem, N. Vanhaecke and G. Meijer. Manipulation and Control of Molecular Beams. Chem. Rev. 112, 4828-4878 (2012).

23. E. Narevicius and M. G. Raizen. Toward Cold Chemistry with Magnetically Decelerated Supersonic Beams. Chem. Rev. 112, 4879-4889 (2012).

24. N. Akerman, M. Karpov, Y. Segev, N. Bibelnik, J. Narevicius and E. Narevicius. Trapping of Molecular Oxygen together with Lithium Atoms. Phys. Rev. Lett. 119, 073204 (2017).

25. Y. Segev, M. Pitzer, M. Karpov, N. Akerman, J. Narevicius and E. Narevicius. Collisions between cold molecules in a superconducting magnetic trap. Nature 572, 189-193 (2019). 
26. Y. Liu, M. Vashishta, P. Djuricanin, S. Zhou, W. Zhong, T. Mittertreiner, D. Carty and T. Momose. Magnetic Trapping of Cold Methyl Radicals. Phys. Rev. Lett. 118, 093201 (2017).

27. J. Riedel, S. Hoekstra, W. Jäger, J.J. Gilijamse, S. Y. T. van de Meerakker and G. Meijer. Accumulation of Stark-decelerated NH molecules in a magnetic trap. Eur. Phys. J. D 65, 161-166 (2011).

28. L. Caldwell, H. J. Williams, N. J. Fitch, J. Aldegunde, J. M. Hutson, B. E. Sauer and M. R. Tarbutt. Long Rotational Coherence Times of Molecules in a Magnetic Trap. Phys. Rev. Lett. 124, 063001 (2020).

29. H. J. Williams, L. Caldwell, N. J. Fitch, S. Truppe, J. Rodewald, E. A. Hinds, B. E. Sauer and M. R. Tarbutt. Magnetic Trapping and Coherent Control of Laser-Cooled Molecules. Phys. Rev. Lett. 120, 163201 (2018).

30. R. Wester. Radiofrequency multipole traps: tools for spectroscopy and dynamics of cold molecular ions. J. Phys. B: At. Mol. Opt. Phys. 42, 154001 (2009).

31. B. R. Heazlewood. Cold ion chemistry within Coulomb crystals. Mol. Phys. 117, 1934-1941 (2019).

32. H. L. Bethlem, F. M. H. Crompvoets, R. T. Jongma, S. Y. T. van de Meerakker, and G. Meijer. Deceleration and trapping of ammonia using time-varying electric fields. Phys. Rev. A 65053416 (2002)

33. S. A. Rangwala, T. Junglen, T. Rieger, P. W. H. Pinkse and G. Rempe. Continuous source of translationally cold dipolar molecules. Phys. Rev. A 67, 043406 (2003).

34. S. Chervenkov, X. Wu, J. Bayerl, A. Rohlfes, T. Gantner, M. Zeppenfeld and G. Rempe. Continuous Centrifuge Decelerator for Polar Molecules. Phys. Rev. Lett. 112, 013001 (2014).

35. M. Zeppenfeld, B. G. U. Englert, R. Glöckner, A. Prehn, M. Mielenz, C. Sommer, L. D. van Buuren, M. Motsch and G. Rempe. Sisyphus cooling of electrically trapped polyatomic molecules. Nature 491, 570-573 (2012).

36. N. Tariq, N. Al Taisan, V. Singh and J. D. Weinstein. Spectroscopic Detection of the LiHe Molecule. Phys. Rev. Lett. 110, 153201 (2013).

37. V. Singh, K. S. Hardman, N. Tariq, M.-J. Lu, A. Ellis, M. J. Morrison and J. D. Weinstein. Chemical Reactions of Atomic Lithium and Molecular Calcium Monohydride at 1 K. Phys. Rev. Lett. 108, 203201 (2012).

38. N. R. Hutzler, H.-I Lu and J. M. Doyle. The Buffer Gas Beam: An Intense, Cold, and Slow Source for Atoms and Molecules. Chem. Rev. 112, 4803-4827 (2012).

39. J. W. Niman, B. S. Kamerin, D. J. Merthe, L. Kranabetter and V. V. Kresin. Oriented Polar Molecules Trapped in Cold Helium Nanodropets: Electrostatic Deflection, Size Separation, and Charge Migration. Phys. Rev. Lett. 123, 043203 (2019).

40. A.Mauracher, O.Echt, A. M. Ellis, S. Yang, D. K. Bohme, J. Postler, A. Kaiser, S. Denifl and P. Scheier. Cold physics and chemistry: Collisions, ionization and reactions inside helium nanodroplets close to zero K. Phys. Rep. 751, 1-90 (2018).

41. A. Slenczka and J. P. Toennies, Chemical dynamics inside superfluid helium nanodroplets at $0.37 \mathrm{~K}$, I. W. M. Smith (Ed.), Low Temperatures and Cold Molecules, Imperial College Press, 345-392 (2008).

42. X. Wu, T. Gantner, M. Zeppenfeld, S. Chervenkov, and G. Rempe. Thermometry of Guided Molecular Beams from a Cryogenic Buffer-Gas Cell ChemPhysChem 17 3631-3640 (2016)

43. M.-G. Hu, Y. Liu, D. D. Grimes, Y.-W. Lin, A. H. Gheorghe, R. Vexiau, N. Boulufa-Maafa, O. Dulieu, T. Rosenband and K.-K. Ni. Direct observation of bimolecular reactions of ultracold KRb molecules. Science 366, 1111-1115 (2019).

44. J. G. Danzl, E. Haller, M. Gustavsson, M. J. Mark, R. Hart, N. Bouloufa, O. Dulieu, H. Ritsch and H.-C. Nägerl. Quantum Gas of Deeply Bound Ground State Molecules. Science 321, 1062-1066 (2008).

45. F. Lang, K. Winkler, C. Strauss, R. Grimm and J. Hecker Denschlag. Ultracold Triplet Molecules in the Rovibrational Ground State. Phys. Rev. Lett. 101133005 (2008).

46. K.-K. Ni, S. Ospelkaus, M. H. G. de Miranda, A. Pe'er, B. Neyenhuis, J. J. Zirbel, S. Kotochigova, P. S. Julienne, D. S. Jin and J. Ye. A High Phase-Space-Density Gas of Polar Molecules. Science 322, 231-235 (2008).

47. T. Takekoshi, L. Reichsöllner, A. Schindewolf, J. M. Hutson, C. R. Le Sueur, O. Dulieu, F. Ferlaino, R. Grimm and H.-C. Nägerl. Ultracold Dense Samples of Dipolar RbCs Molecules in the Rovibrational and Hyperfine Ground State. Phys. Rev. Lett. 113, 205301 (2014).

48. P. K. Molony, P. D. Gregory, Z. Ji, B. Lu, M. P. Köppinger, C. R. Le Sueur, C. L. Blackley, J. M. Hutson and S. L. Cornish. Creation of Ultracold ${ }^{87} \mathrm{Rb}^{133} \mathrm{Cs}$ Molecules in the Rovibrational Ground State. Phys. Rev. Lett. 113, 255301 (2014).

49. J. W. Park, S. A. Will and M. W. Zwierlein. Ultracold Dipolar Gas of Fermionic ${ }^{23} \mathrm{Na}^{40} \mathrm{~K}$ Molecules in Their Absolute Ground State.Phys. Rev. Lett. 114, 205302 (2015). 
50. M. Guo, B. Zhu, B. Lu, X. Ye, F. Wang, R. Vexiau, N. Bouloufa-Maafa, G. Quéméner, O. Dulieu and D. Wang. Creation of an Ultracold Gas of Ground-State Dipolar ${ }^{23} \mathrm{Na}^{87} \mathrm{Rb}$ Molecules. Phys. Rev. Lett. 116, 205303 (2016).

51. E. L. Raab, M. Prentiss, A. Cable, S. Chu and D. E. Pritchard. Trapping of Neutral Sodium Atoms with Radiation Pressure. Phys. Rev. Lett. 59, 2631-2634 (1987).

52. J. F. Barry, D. J. McCarron, E. B. Norrgard, M. H. Steinecker and D. DeMille. Magneto-optical trapping of a diatomic molecule. Nature 512, 286-289 (2014).

53. H. J. Williams, S. Truppe, M. Hambach, L. Caldwell, N. J. Fitch, E. A. Hinds, B. E. Sauer and M. R. Tarbutt. Characteristics of a magneto-optical trap of molecules. New J. Phys. 19113035 (2017).

54. K. M. Jones, E. Tiesinga, P. D. Lett and P. S. Julienne. Ultracold photoassociation spectroscopy: Long-range molecules and atomic scattering. Rev. Mod. Phys. 78, 483-535 (2006).

55. C. Chin, R. Grimm, P. S. Julienne and E. Tiesinga. Feshbach resonances in ultracold gases. Rev. Mod. Phys. 82, 1225-1286 (2010).

56. J. Toscano, C. J. Rennick, T. P. Softley and B. R. Heazlewood. A magnetic guide to purify radical beams. J. Chem. Phys. 149, 174201 (2018).

57. K. A. E. Meyer, L. L. Pollum, L. S. Petralia, A. Tauschinsky, C. J. Rennick, T. P. Softley and B. R. Heazlewood. Ejection of Coulomb crystals from a linear Paul ion trap for ion-molecule reaction studies. J. Phys. Chem. A 119, 12449-12456 (2015).

58. L. Anderegg, L. W. Cheuk, Y. Bao, S. Burchesky, W. Ketterle, K.-K. Ni and J. M. Doyle. An optical tweezer array of ultracold molecules. Science 365, 1156-1158 (2019).

59. A. T. Grier, M. Cetina, F. Oručević and V. Vuletić. Observation of Cold Collisions between Trapped Ions and Trapped Atoms. Phys. Rev. Lett. 102, 223201 (2009).

60. M. Tomza, K. Jachymski, R. Gerritsma, A. Negretti, T. Calarco, Z. Idziaszek and P. S. Julienne. Cold hybrid ion-atom systems. Rev. Mod. Phys. 91, 035001 (2019).

61. W. Phillips. Laser cooling and trapping of neutral atoms. Rev. Mod. Phys. 70 721-741 (1988)

62. E. S. Shuman, J. F. Barry and D. DeMille. Laser cooling of a diatomic molecule. Nature 467, 820-823 (2010).

63. V. Zhelyazkova, A. Cournol, T. E. Wall, A. Matsushima, J. J. Hudson, E. A. Hinds, M. R. Tarbutt and B. E. Sauer. Laser cooling and slowing of CaF molecules. Phys. Rev. A 89, 053416 (2014).

64. I. Kozyryev, L. Baum, K. Matsuda, B. L. Augenbraun, L. Anderegg, A. P. Sedlack and J. M. Doyle. Sisyphus Laser Cooling of a Polyatomic Molecule. Phys. Rev. Lett. 118, 173201 (2017).

65. J. Lim, J. R. Almond, M. A. Trigatzis, J. A. Devlin, N. J. Fitch, B. E. Sauer, M. R. Tarbutt and E. A. Hinds. Laser Cooled YbF Molecules for Measuring the Electron's Electric Dipole Moment. Phys. Rev. Lett. 120, 123201 (2018).

66. M. R. Tarbutt. Laser cooling of molecules. Contemp. Phys. 59, 356-376 (2019).

67. S. Ding, Y. Wu, I. A. Finneran, J. J. Burau and J. Ye. Sub-Doppler Cooling and Compressed Trapping of YO Molecules at $\mu \mathrm{K}$ Temperatures. Phys. Rev. X 10, 021049 (2020).

68. D. Mitra, N. B. Vilas, C. Hallas, L. Anderegg, B. L. Augenbraun, L. Baum, C. Miller, S. Raval and J. M. Doyle. Direct Laser Cooling of a Symmetric Top Molecule. arXiv:2004.02848v2 (2020).

69. M. T. Hummon, T. V. Tscherbul, J. Kłos, H.-I Lu, E. Tsikata, W. C. Campbell, A. Dalgarno and J. M. Doyle. Cold N + NH Collisions in a Magnetic Trap. Phys. Rev. Lett. 106, 053201 (2011).

70. L. P. Parazzoli, N. J. Fitch, P. S. Żuchowski, J. M. Hutson and H. J. Lewandowski. Large Effects of Electric Fields on Atom-Molecule Collisions at Millikelvin Temperatures. Phys. Rev. Lett. 106, 193201 (2011).

71. H. Son, J. J. Park, W. Ketterle and A. O. Jamison. Collisional cooling of ultracold molecules. Nature 580, 197-200 (2020).

72. G. Valtolina, K. Matsuda, W. G. Tobias, J.-R. Li, L. De Marco and J. Ye. Dipolar evaporation of reactive molecules to below the Fermi temperature. arXiv:2007.12277v1 (2020).

73. M. T. Bell and T. P. Softley. Ultracold Molecules and Ultracold Chemistry. Molecular Physics 107, 99-132 (2009).

74. D. C. Clary and J. P. Henshaw. Chemical reactions dominated by long-range intermolecular forces. Faraday Discuss. Chem. Soc. 84 333-349 (1987).

75. D. C. Clary. Rate Constants for the Reactions of Ions with Dipolar Polyatomic Molecules. J. Chem. Soc., Faraday Trans. 2 83, 139-148 (1987). 
76. J. Troe. Statistical adiabatic channel model for ion-molecule capture processes. J. Chem. Phys. 87, 2773-2780 (1987).

77. E. I. Dashevskaya, A. I. Maergoiz, J. Troe, I. Litvin and E. E. Nikitin. Low-temperature behavior of capture rate constants for inverse power potentials. J. Chem. Phys. 118, 7313-7320 (2003).

78. R. Kosloff and D. Kosloff. Absorbing Boundaries for Wave Propagation Problems J. Comput. Phys. 63, 363-376 (1986).

79. N. Balakrishnan. Perspective: Ultracold molecules and the dawn of cold controlled chemistry. J. Chem. Phys. 145, 150901 (2016).

80. Z. Idziaszek and P. S. Julienne. Universal Rate Constants for Reactive Collisions of Ultracold Molecules. Phys. Rev. Lett. 104, 113202 (2010).

81. P. D. Gregory, M. D. Frye, J. A. Blackmore, E. M. Bridge, R. Sawant, J. M. Hutson and S. L. Cornish. Sticky collisions of ultracold RbCs molecules. Nat. Commun. 10, 3104 (2019).

82. C. Berteloite, M. Lara, A. Bergeat, S. D. Le Picard, F. Dayou, K. M. Hickson, A. Canosa, C. Naulin, J.-M. Launay, I. R. Sims and M. Costes. Kinetics and Dynamics of the $\mathrm{S}\left({ }^{1} \mathrm{D}_{2}\right)+\mathrm{H}_{2} \rightarrow \mathrm{SH}+\mathrm{H}$ Reaction at Very Low Temperatures and Collision Energies. Phys. Rev. Lett. 105, 203201 (2010).

83. S. Chefdeville, T. Stoecklin, A. Bergeat, K. M. Hickson, C. Naulin and M. Costes. Appearance of Low Energy Resonances in CO-Para- $\mathrm{H}_{2}$ Inelastic Collisions. Phys. Rev. Lett. 109, 023201 (2012).

84. S. Chefdeville, Y. Kalugina, S. Y. T. van de Meerakker, C. Naulin, F. Lique and M. Costes. Observation of Partial Wave Resonances in Low-Energy $\mathrm{O}_{2}-\mathrm{H}_{2}$ Inelastic Collisions. Science 341, 1094-1096 (2013).

85. S. N. Vogels, J. Onvlee, S. Chefdeville, A. van der Avoird, G. C. Groenenboom and S. Y. T. van de Meerakker. Imaging resonances in low-energy NO-He inelastic collisions. Science 350, 787-790 (2015).

86. S. N. Vogels, T. Karman, J. Kłos, M. Besemer, J. Onvlee, A. van der Avoird, G. C. Groenenboom and S. Y. T. van de Meerakker. Scattering resonances in bimolecular collisions between $\mathrm{NO}$ radicals and $\mathrm{H}_{2}$ challenge the theoretical gold standard. Nat. Chem. 10, 435-440 (2018).

87. T. Yang, L. Huang, C. Xiao, J. Chen, T. Wang, D. Dai, F. Lique, M. H. Alexander, Z. Sun, D. H. Zhang, X. Yang and D. M. Neumark. Enhanced reactivity of fluorine with para-hydrogen in cold interstellar clouds by resonance-induced quantum tunnelling. Nat. Chem. 11, 774-781 (2019).

88. A. Klein, Y. Shagam, W. Skomorowski, P. S. Żuchowski, M. Pawlak, L. M. C. Janssen, N. Moiseyev, S. Y. T. van de Meerakker, A. van der Avoird, C. P. Koch and E. Narevicius. Directly probing anisotropy in atom-molecule collisions through quantum scattering resonances. Nat. Phys. 13, 35-38 (2017).

89. T. de Jongh, M. Besemer, Q. Shuai, T. Karman, A. van der Avoird, G. C. Groenenboom and S. Y. T. van de Meerakker. Imaging the onset of the resonance regime in low-energy NO-He collisions. Science 368, 626-630 (2020).

90. J. Marquette, B. Rowe, G. Dupeyrat, G. Poissant and C. Rebrion. Ion-polar-molecule reactions: A CRESU study of $\mathrm{He}^{+}$, $\mathrm{C}^{+}, \mathrm{N}^{+}+\mathrm{H}_{2} \mathrm{O}, \mathrm{NH}_{3}$ at 27,68 and $163 \mathrm{~K}$ Chem. Phys. Lett. 122, 431-435 (1985).

91. E. Herbst and T. J. Millar. The Chemistry of Cold Interstellar Cloud Cores in 'Low Temperatures and Cold Molecules' Ed. I. W. M. Smith, Imperial College Press, Chapter 1, (2008)

92. A. Canosa, F. Goulay, I. R. Sims and B. R. Rowe. Gas Phase Reactive Collisions at Very Low Temperature: Recent Experimental Advacnes and Perspectives in 'Low Temperatures and Cold Molecules' Ed. I. W. M. Smith, Imperial College Press, Chapter 2, (2008)

93. S. J. Klippenstein and Y. Georgievskii. Theory of Low Temperature Gas-Phase reactions in 'Low Temperatures and Cold Molecules' Ed. I. W. M. Smith, Imperial College Press, Chapter 4, (2008)

94. B. Rowe, A. Canosa and V. Le Page. FALP and CRESU studies of ionic reactions. Int. J. Mass Spectrom. Ion Proc. 149-150, 573-596 (1995).

95. I. W. M. Smith. Laboratory Astrochemistry: Gas-Phase Processes. Annu. Rev. Astron. Astrophys. 49, 29-66 (2011).

96. R. J. Shannon, M. A. Blitz, A. Goddard and D. E. Heard. Accelerated chemistry in the reaction between the hydroxyl radical and methanol at interstellar temperatures facilitated by tunnelling. Nat. Chem. 5, 745-749 (2013).

97. M. Tizniti, S. D. Le Picard, F. Lique, C. Berteloite, A. Canosa, M. H. Alexander and I. R. Sims. The rate of the F $+\mathrm{H}_{2}$ reaction at very low temperatures. Nat. Chem. 6, 141-145 (2014).

98. K. J. Catani, J. Greenberg, B. V. Saarel and H. J. Lewandowski. Reactions of translationally cold trapped $\mathrm{CCl}^{+}$with acetylene $\left(\mathrm{C}_{2} \mathrm{H}_{2}\right)$. J. Chem. Phys. 152, 234310 (2020). 
99. R. Plašil, T. Mehner, P. Dohnal, T. Kotrík, J. Glosík and D. Gerlich. Reactions of cold trapped $\mathrm{CH}^{+}$ions with slow H atoms. Astrophys. J. 737, 60 (2011).

100. D. Mulin, Š. Roučka, P. Jusko, I. Zymak, R. Plašil, D. Gerlich, R. Wester and J. Glosík. H/D exchange in reactions of $\mathrm{OH}^{-}$with $\mathrm{D}_{2}$ and of $\mathrm{OD}^{-}$with $\mathrm{H}_{2}$ at low temperatures. Phys. Chem. Chem. Phys. 17, 8732-8739 (2015).

101. D. Hauser, S. Lee, F. Carelli, S. Spieler, O. Lakhmanskaya, E. S. Endres, S. S. Kumar, F. Gianturco and Roland Wester. Rotational state-changing cold collisions of hydroxyl ions with helium. Nat. Phys. 11, 467-470 (2015).

102. T. D. Tran, S. Rednyk, A. Kovalenko, Š. Roučka, P. Dohnal, R. Plašil, D. Gerlich and J. Glosík. Formation of $\mathrm{H}_{2} \mathrm{O}^{+}$and $\mathrm{H}_{3} \mathrm{O}^{+}$Cations in Reactions of $\mathrm{OH}^{+}$and $\mathrm{H}_{2} \mathrm{O}^{+}$with $\mathrm{H}_{2}$ : Experimental Studies of the Reaction Rate Coefficients from $\mathrm{T}=$ 15 to 300 K. Astrophys. J. 85425 (2018).

103. A. Bergeat, A. Faure, S. B. Morales, A. Moudens and C. Naulin. Low-Energy Water-Hydrogen Inelastic Collisions. J. Phys. Chem. A 124, 259-264 (2020).

104. T. J. Millar. Deuterium Fractionation in Interstellar Clouds. Space Sci. Rev. 106, $73-86$ (2003).

105. E. Herbst. Isotopic Fractionation by Ion-Molecule Reactions. Space Sci. Rev. 106, 293-304 (2003).

106. L. S. Petralia, A. Tsikritea, J. Loreau, T. P. Softley and B. R. Heazlewood. Strong inverse kinetic isotope effect observed in ammonia charge exchange reactions. Nat. Commun. 11, 173 (2020).

107. Y.-P. Chang, K. Długołęcki, J. Küpper, D. Rösch, D. Wild and S. Willitsch. Specific Chemical Reactivities of Spatially Separated 3-Aminophenol Conformers with Cold $\mathrm{Ca}^{+}$Ions. Science 342 98-101 (2013).

108. A. Kilaj, H. Gao, D. Rösch, U. Rivero, J. Küpper and S. Willitsch. Observation of different reactivities of para and ortho-water towards trapped diazenylium ions. Nat. Commun. 9, 2096 (2018).

109. S. Willitsch, M. T. Bell, A. D. Gingell and T. P. Softley. Chemical applications of laser- and sympathetically-cooled ions in ion traps. Phys. Chem. Chem. Phys. 10, 7200-7210 (2008).

110. M. T. Bell, A. D. Gingell, J. M. Oldham, T. P. Softley and S. Willitsch. Ion-molecule chemistry at very low temperatures: cold chemical reactions between Coulomb-crystallized ions and velocity-selected neutral molecules. Faraday Discuss. 142, 73-91 (2009).

111. S. Willitsch. Coulomb-crystallised molecular ions in traps: methods, applications, prospects. Int. Rev. Phys. Chem. 3, 175-199 (2012).

112. R. C. Thompson. Ion Coulomb crystals. Contemp. Phys. 56, 63-79 (2015).

113. M. Drewsen. Ion Coulomb crystals. Phys. B: Condens. Matter 460, 105-113 (2015).

114. D. Zhang and S. Willitsch. Cold Chemistry: Molecular Scattering and Reactivity Near Absolute Zero, RSC Publishing (2017).

115. S. Willitsch. Chemistry with Controlled Ions. Adv. Chem. Phys. 162, 307-340 (2017).

116. A. T. Calvin and K. R. Brown. Spectroscopy of Molecular Ions in Coulomb Crystals. J. Phys. Chem. Lett. 9, 5797-5804 (2018).

117. T. Yang, A. Li, G. K. Chen, C. Xie, A. G. Suits, W. C. Campbell, H. Guo and E. R. Hudson. Optical Control of Reactions between Water and Laser-Cooled Be ${ }^{+}$Ions. J. Phys. Chem. Lett. 9, 3555-3560 (2018).

118. J. Greenberg, P. C. Schmid, M. Miller, J. F. Stanton and H. J. Lewandowski. Quantum-state-controlled reactions between molecular radicals and ions. Phys. Rev. A 98, 032702 (2018).

119. P. C. Schmid, M. I. Miller, J. Greenberg, T. L. Nguyen, J. F. Stanton and H. J. Lewandowski. Quantum-state-specific reaction rate measurements for the photo-induced reaction $\mathrm{Ca}^{+}+\mathrm{O}_{2} \rightarrow \mathrm{CaO}^{+}+\mathrm{O}$. Mol. Phys. 117 3036-3042 (2019).

120. B. R. Heazlewood. Cold ion chemistry within Coulomb crystals. Mol. Phys. 117, 1934-1941 (2019).

121. M. Lara, S. Chefdeville, K. M. Hickson, A. Bergeat, C. Naulin, J.-M. Launay and M. Costes. Dynamics of the $S\left({ }^{1} D_{2}\right)+$ $\mathrm{HD}(j=0)$ Reaction at Collision Energies Approaching the Cold Regime: A Stringent Test for Theory. Phys. Rev. Lett. 109, 133201 (2012).

122. E. Lavert-Ofir, Y. Shagam, A. B. Henson, S. Gersten, J. Kłos, P. S. Zuchowski, J. Narevicius and E. Narevicius. Observation of the isotope effect in sub-kelvin reactions. Nat. Chem. 6, 332-335 (2014).

123. Y. Shagam, A. Klein, W. Skomorowski, R. Yun, V. Averbukh, C. P. Koch and E. Narevicius. Molecular hydrogen interacts more strongly when rotationally excited at low temperatures leading to faster reactions. Nat. Chem. 7, 921-926 (2015). 
124. S. D. S. Gordon, J. J. Omiste, J. Zou, S. Tanteri, P. Brumer and A. Osterwalder. Quantum-state-controlled channel branching in cold $\mathrm{Ne}\left({ }^{3} \mathrm{P}_{2}\right)+$ Ar chemi-ionization. Nat. Chem. 10, 1190-1195 (2018).

125. P. Allmendinger, J. Deiglmayr, O. Schullian, K. Hoveler, J. A. Agner, H. Schmutz and F. Merkt. Chem. Phys. Chem. 17, 3596-3608 (2016).

126. P. Allmendinger, J. Deiglmayr, K. Hoveler, O. Schullian and F. Merkt. Observation of enhanced rate coefficients in the $\mathrm{H}_{2}^{+}+\mathrm{H}_{2} \rightarrow \mathrm{H}_{3}^{+}+\mathrm{H}$ reaction at low collision energies. J. Chem. Phys. 145, 244316 (2016).

127. R. Saito, S. Haze, M. Sasakawa, R. Nakai, M. Raoult, H. Da Silva, Jr., O. Dulieu and T. Mukaiyama. Characterization of charge-exchange collisions between ultracold ${ }^{6} \mathrm{Li}$ atoms and ${ }^{40} \mathrm{Ca}^{+}$ions. Phys. Rev. A 95, 032709 (2017).

128. P. Puri, M. Mills, I. Simbotin, J. A. Montgomery Jr., R. Côté, C. Schneider, A. G. Suits and E. R. Hudson. Reaction blockading in a reaction between an excited atom and a charged molecule at low collision energy. Nat. Chem. 11 615-621 (2019).

129. A. D. D. Dörfler, P. Eberle, D. Koner, M. Tomza, M. Meuwly and S. Willitsch. Long-range versus short-range effects in cold molecular ion-neutral collisions. Nat. Commun. 10, 5429 (2019).

130. V. Singh, K. S. Hardman, N. Tariq, M.-J. Lu, A. Ellis, M. J. Morrison, and J. D. Weinstein, Chemical reactions of atomic lithium and molecular calcium monohydride at 1 K. Phys. Rev. Lett. 108, 203201 (2012).

131. B. K. Stuhl, M. T. Hummon and J. Ye. Cold State-Selected Molecular Collisions and Reactions. Annu. Rev. Phys. Chem. 65, 501-518 (2014).

132. J. Toscano, H. Lewandowski and B. R. Heazlewood. Cold and controlled chemical reaction dynamics. Phys. Chem. Chem. Phys. 22, 9180-9194 (2020).

133. B. K. Kendrick and N. Balakrishnan. Geometric Phase Effects in Ultracold Chemical Reactions. Atoms 7, 65 (2019).

134. J. Hazra, B. K. Kendrick and N. Balakrishnan. Importance of Geometric Phase Effects in Ultracold Chemistry. J. Phys. Chem. A 119, 12291-12303 (2015).

135. T. Feldker, H. Fürst, H. Hirzler, N. V. Ewald, M. Mazzanti, D. Wiater, M. Tomza and R. Gerritsma. Buffer gas cooling of a trapped ion to the quantum regime. Nat. Phys. 16, 413-416 (2020).

136. C. Sias. Making ions cooler. Nat. Phys. 16, 378-379 (2020).

137. S. Ospelkaus, K.-K. Ni, D. Wang, M. H. G. de Miranda, B. Neyenhuis, G. Quéméner, P. S. Julienne, J. L. Bohn, D. S. Jin and J. Ye. Quantum-state controlled chemical reactions of ultracold potassium-rubidium molecules. Science 327, 853-857 (2010).

138. K.-K. Ni, S. Ospelkaus, D. Wang, G. Quéméner, B. Neyenhuis, M. H. G. de Miranda, J. L. Bohn, J. Ye and D. S. Jin. Dipolar collisions of polar molecules in the quantum regime. Nature 464, 1324-1328 (2010).

139. L. D. Marco, G. Valtolina, K. Matsuda, W. G. Tobias, J. P. Covey, J. Ye. A fermi degenerate gas of polar molecules. Science 363, 853-856 (2018).

140. T. M. Rvachov, H. Son, A. T. Sommer, S. Ebadi, J. J. Park, M. W. Zwierlein, W. Ketterle and A. O. Jamison. Long-lived ultracold molecules with electric and magnetic dipole moments. Phys. Rev. Lett. 119, 143001 (2017).

141. B. Drews, M. Deiß, K. Jachymksi, Z. Idziaszek, and J. Hecker Denschlag. Inelastic collisions of ultracold triplet $\mathrm{Rb}_{2}$ molecules in the rovibrational ground state. Nat. Commun. 8, 14854 (2017).

142. D. K. Hoffmann, T. Paintner, W. Limmer, D. S. Petrov and J. Hecker Denschlag. Reaction kinetics of ultracold moleculemolecule collisions. Nat. Commun. 9, 5244 (2018).

143. X. Ye, M. Guo, M. L. González-Martínez, G. Quéméner and D. Wang. Collisions of ultracold ${ }^{23} \mathrm{Na}^{87} \mathrm{Rb}$ molecules with controlled chemical reactivities. Sci. Adv. 4, eaaq0083 (2018).

144. M. Guo, X. Ye, J. He, M. L. González-Martínez, R. Vexiau, G. Quéméner and D. Wang. Dipolar Collisions of Ultracold Ground-State Bosonic Molecules. Phys. Rev. X 8, 041044 (2018).

145. H. Yang, D.-C. Zhang, L. Liu, Y.-X. Liu, J. Nan, B. Zhao and J.-W. Pan. Observation of magnetically tunable Feshbach resonances in ultracold ${ }^{23} \mathrm{Na}^{40} \mathrm{~K}+{ }^{40} \mathrm{~K}$ collisions. Science $363261-264$ (2019).

146. Z. Z. Yan, J. W. Park, Y. Ni, H. Loh, S. Will, T. Karman and M. Zwierlein. Resonant Dipolar Collisions of Ultracold Molecules Induced by Microwave Dressing. Phys. Rev. Lett. 125, 063401 (2020).

147. P. D. Gregory, J. A. Blackmore, S. L. Bromley and S. L. Cornish. Loss of ultracold ${ }^{87} \mathrm{Rb}^{133} \mathrm{Cs}$ molecules via optical excitation of long-lived two-body collision complexes. Phys. Rev. Lett. 124, 163402 (2020). 
148. Y. Liu, M.-G. Hu, M. A. Nichols, D. D. Grimes, T. Karman, H. Guo and K.-K. Ni. Photo-excitation of long-lived transient intermediates in ultracold reactions. Nat. Phys. https://doi.org/10.1038/s41567-020-0968-8 (2020).

149. Y. Liu, D. D. Grimes, M.-G. Hu and K.-K. Ni. Probing ultracold chemistry using ion spectrometry. Phys. Chem. Chem. Phys. 22, 4861-4874 (2020).

150. M.-G. Hu, Y. Liu, M. A. Nichols, L. Zhu, G. Quéméner, O. Dulieu and K.-K. Ni. Product-state control of ultracold reactions via conserved nuclear spins. arXiv:2005.10820v1 (2020).

151. M. Quack. Detailed Symmetry Selection Rules for Molecular Collisions. Molecular Physics 34, 477 (1977).

152. K. Dulitz, T. Sixt, J. Guan, J. Grzesiak, M. Debatin and F. Stienkemeier. Suppression of Penning ionization by orbital angular momentum conservation. Phys. Rev. A 102, 022818 (2020).

153. J. Wolf, M. Deiß, A. Krükow, E. Tiemann, B. P. Ruzic, Y. Wang, J. P. D’Incao, P. S. Julienne, J. Hecker Denschlag. State-to-state chemistry for three-body recombination in an ultracold rubidium gas. Science 358, 921-924 (2017).

154. M. H. G. de Miranda, A. Chotia, B. Neyenhuis, D. Wang, G. Quéméner, S. Ospelkaus, J. L. Bohn, J. Ye and D. S. Jin. Controlling the quantum stereodynamics of ultracold bimolecular reactions. Nat. Phys. 7 502-507 (2011).

155. J. F. E. Croft, C. Makrides, M. Li, A. Petrov, B. K. Kendrick, N. Balakrishnan and S. Kotochigova. Universality and chaoticity in ultracold K + KRb chemical reactions. Nat. Commun. 8, 15897 (2017).

156. L. W. Cheuk, L. Anderegg, Y. Bao, S. Burchesky, S. S. Yu, W. Ketterle, K.-K. Ni and J. M. Doyle. Observation of Collisions between Two Ultracold Ground-State CaF Molecules. Phys. Rev. Lett. 125, 043401 (2020).

157. M. Karpov, M. Pitzer, Y. Segev, J. Narevicius and E. Narevicius. Low-energy collisions between carbon atoms and oxygen molecules in a magnetic trap. arXiv:2006.08388v1 (2020). 